\title{
Simple-to-Apply Wetting Model to Predict Thermodynamically Stable and Metastable Contact Angles on Textured/Rough/Patterned Surfaces
}

Yair Kaufman, ${ }^{*}, \dagger, \downarrow, \triangle \odot$ Szu-Ying Chen, ${ }^{\dagger, \triangle}$ ○ Himanshu Mishra, ${ }^{\S, \|}$ Alex M. Schrader, ${ }^{\dagger}$ Dong Woog Lee, ${ }^{\perp}$ Saurabh Das, ${ }^{\dagger}$ Stephen H. Donaldson, Jr., ${ }^{\dagger, \#}$ and Jacob N. Israelachvili ${ }^{\dagger, \S, \text { II }}$

${ }^{\dagger}$ Department of Chemical Engineering, ${ }^{\S}$ California NanoSystems Institute, and ${ }^{\text {IT }}$ Materials Department, University of California at Santa Barbara, Santa Barbara, California 93106, United States

${ }^{\ddagger}$ Zuckerberg Institute for Water Research, The Jacob Blaustein Institutes for Desert Research, Ben-Gurion University of the Negev, Sede Boqer Campus, 84990 Midreshet Ben-Gurion, Israel

"Water Desalination and Reuse Center, Biological and Environmental Science and Engineering Division, King Abdullah University of Science and Technology, Thuwal 23955-6900, Saudi Arabia

${ }^{\perp}$ School of Energy and Chemical Engineering, Ulsan National Institute of Science and Technology (UNIST), UNIST-gil 50, Ulsan 689-798, Republic of Korea

\#Département de Physique, Ecole Normale Supérieure, PSL Research University, CNRS, 24 rue Lhomond 75005 Paris, France

\section{Supporting Information}

ABSTRACT: Rough/patterned/textured surfaces with nano/microcavities that broaden below the surface-known as "re-entrants"-can be omniphobic (macroscopic contact angle greater than $90^{\circ}$ for both water and oils). The existing theoretical models that explain the effects of texture on wetting are complex and do not provide a simple procedure for predicting the thermodynamically stable and metastable states and their corresponding contact angles (for example, wetting states that involve partially filled cavities). Here, we develop a simple-to-apply wetting model that allows for (1) predicting a priori the wetting state (partially or fully filled) of the cavities both under and outside the liquid droplet and the corresponding macroscopic

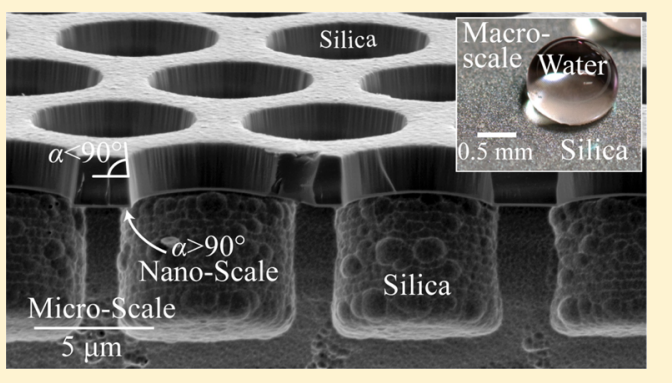
contact angles on any type of textured surface; (2) determining the conditions under which metastable states exist; and (3) engineering specific nano/microtextures that yield any desired macroscopic contact angle, $\theta_{\mathrm{t}}$, for a given intrinsic contact angle $\theta_{0}$. Subsequently, we experimentally demonstrate how one can use the model to predict the metastable and the thermodynamically stable contact angles on nondeformable textured surfaces consisting of arrays of axisymmetric cavities/protrusions. In this model, we do not consider the effects of gravitational forces, Laplace pressure of the droplet, line tension, droplet impact velocity, and quantitative aspects of contact angle hysteresis. Nonetheless, the model is suitable for accurately predicting the contact angles of macroscopic droplets (droplet volume $\sim 1 \mu \mathrm{L}$ and base diameters $<2 \mathrm{~mm}$ ), which is of immense relevance in engineering. In the experimental section we also discuss the suitability of the model to be extended in order to include the effects of contact angle hysteresis on the macroscopic apparent contact angle on textured surfaces. Controlling these macroscopic contact angles, whether higher or lower than the intrinsic angle, $\theta_{0}$, is desirable for many applications including nonwetting, self-cleaning, and antifouling surfaces and for completely wetting/spreading applications, such as creams, cosmetics, and lubricant fluids.

\section{INTRODUCTION}

The effects of nano- and micro-scale roughness/texture on the wetting of solids have been studied extensively in recent years. $^{1-28}$ Classic models of wetting, e.g., the Young, ${ }^{29}$ Wenzel, $^{30}$ and Cassie-Baxter ${ }^{38}$ models, which are elaborated below, are commonly used to rationalize experimental observations, usually a posteriori rather than a priori. The Young equation (Figure 1A) describes the equilibrium contact angle on atomically smooth, flat, and nondeformable surfaces. The Wenzel (Figure 1B) and the Cassie-Baxter (Figure 1C) equations describe two possible wetting states on textured surfaces, where the cavities/pores under the liquid are fully or partially filled, respectively. However, the major limitation of these models is that they do not predict the wetting state (i.e., fully or partially filled) and its stability (metastable or thermodynamically stable) even when the geometric details of the texture are known.

Received: January 2, 2017

Revised: February 18, 2017

Published: February 21, 2017 


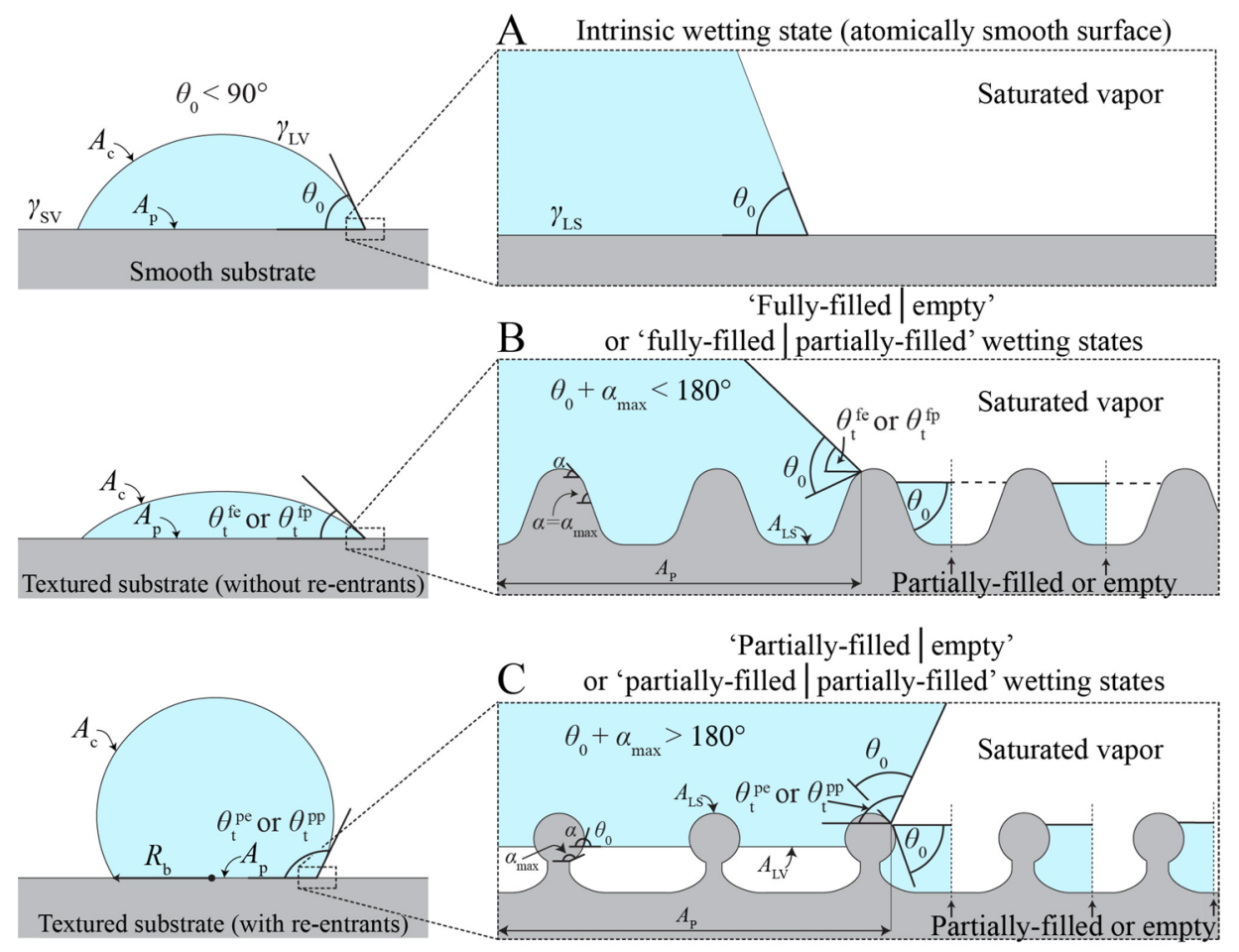

Figure 1. When a liquid droplet is deposited on an atomically smooth and flat solid in the presence of vapor, as depicted in Panel A, a thermodynamically stable contact angle, $\theta_{0}$, is formed, which is given by the Young equation. If a droplet of the same liquid is deposited on a textured/patterned/rough surface made up of the same material, the liquid can fully or partially fill the cavities/pores under the droplet, as depicted in Panels B and C, respectively. In addition, the cavities outside the droplet can be partially filled by condensation as well. Each wetting state-"fully filled I empty" (cavities under the droplet are fully filled, and cavities outside the droplet are empty), "partially filled I empty", "partially filled I partially filled", or "fully filled I partially filled"-yields a different macroscopic contact angle, $\theta_{\mathrm{v}}$ on textured/patterned/rough surfaces (ref 37 describes the wetting states and their corresponding contact angles). Note: the cavities can be connected or unconnected to adjacent cavities. Note also that in Panel C the effects of gravity and the Laplace pressure of the droplet are neglected for $\sim 1 \mu \mathrm{L}$ droplets $(\sim 500 \mu \mathrm{m}$ base radius $)$; therefore, the liquid-vapor interface is drawn flat (see Section 1 in the Supporting Information).

The novelty of the model presented here is the simplicity to apply it to arrays of nano/microcavities/protrusions and other commonly studied textured geometries. Given the richness of this field, other theoretical ways to analyze wetting, such as noncontinuum molecular simulations, ${ }^{31}$ are available; however, the model we present here is significantly easier to apply, predict, and interpret experimental data and can be extended to more complex dynamic behavior on realistic systems. Analytical continuum models that can predict the metastable and thermodynamically stable states and contact angles on any textured surface have been proposed, ${ }^{2,8,22,32-35}$ but those models require extensive mathematical manipulations in order to predict the contact angle. Furthermore, those models do not allow for deriving general conclusions-that is, each texture must be studied separately. The model derived here also provides some important general conclusions for nondeformable axisymmetric cavities/protrusions of any shape (see Summary and Conclusions section).

Starting with the simplest case, when a drop of liquid is placed on a flat and smooth surface and the system is allowed to reach thermodynamic equilibrium under saturated vapor, an intrinsic contact angle, $\theta_{0}$, at the liquid-solid-vapor triple line is formed (see Figure 1A). $\theta_{0}$ can be accurately calculated via Young's equation (discussed below). ${ }^{29}$ We note that throughout the manuscript "Vapor" can be replaced by any other fluid, "Phase 2", as long as "Phase 1" and "Phase 2" are immiscible. ${ }^{36}$

On textured/patterned/rough surfaces, however, the macroscopic contact angle, $\theta_{\mathrm{t}}$ (the subscript " $\mathrm{t}$ " stands for "textured") can be significantly different from $\theta_{0} \cdot{ }^{34}$ Four different extreme wetting states are possible, and their corresponding contact angles are denoted as $\theta_{\mathrm{t}}^{\mathrm{fe}}, \theta_{\mathrm{t}}^{\mathrm{pe}}, \theta_{\mathrm{t}}^{\mathrm{fp}}$, and $\theta_{\mathrm{t}}^{\mathrm{pp}}$ (Figure 1, Panels B and $C$ ), where throughout this paper, we distinguish between the conditions of the cavities under the droplet from those outside the droplet by a vertical line, e.g., "partially filled I empty", also denoted by superscript "pe". Reference 37 and Figure 3 define the nomenclature of these four different wetting states in more detail. If the liquid fully fills the cavities under the droplet and the cavities outside the droplet remain empty (i.e., filled with vapor, see Figure 1B), the Wenzel model is employed to calculate $\theta_{\mathrm{t}}^{\mathrm{fe}}$. The superscript "fe" stands for "fully filled I empty" wetting state, where the cavities under the droplet are fully filled with liquid and the cavities outside the droplet are empty of liquid. ${ }^{30}$ On the other hand, if the liquid partially fills the cavities under the droplet (Figure 1C) and the cavities outside the droplet are empty, the Cassie-Baxter model is invoked to calculate $\theta_{\mathrm{t}}^{\text {pe }}$ ("pe" stands for "partially filled I empty"). ${ }^{38,39}$ It is important to note that the Wenzel and Cassie-Baxter models implicitly assume that the cavities outside the droplet are empty (filled with vapor), and their state (whether they are empty or partially filled with liquid) does not affect the macroscopic contact angle, $\theta_{\mathrm{t}}$. This assumption implies that the state outside the droplet is not necessarily in thermodynamic equilibrium with the vapor phase outside the droplet.

Nonetheless, the Wenzel and Cassie-Baxter models show that the "fully filled I empty" and the "partially filled I empty" 

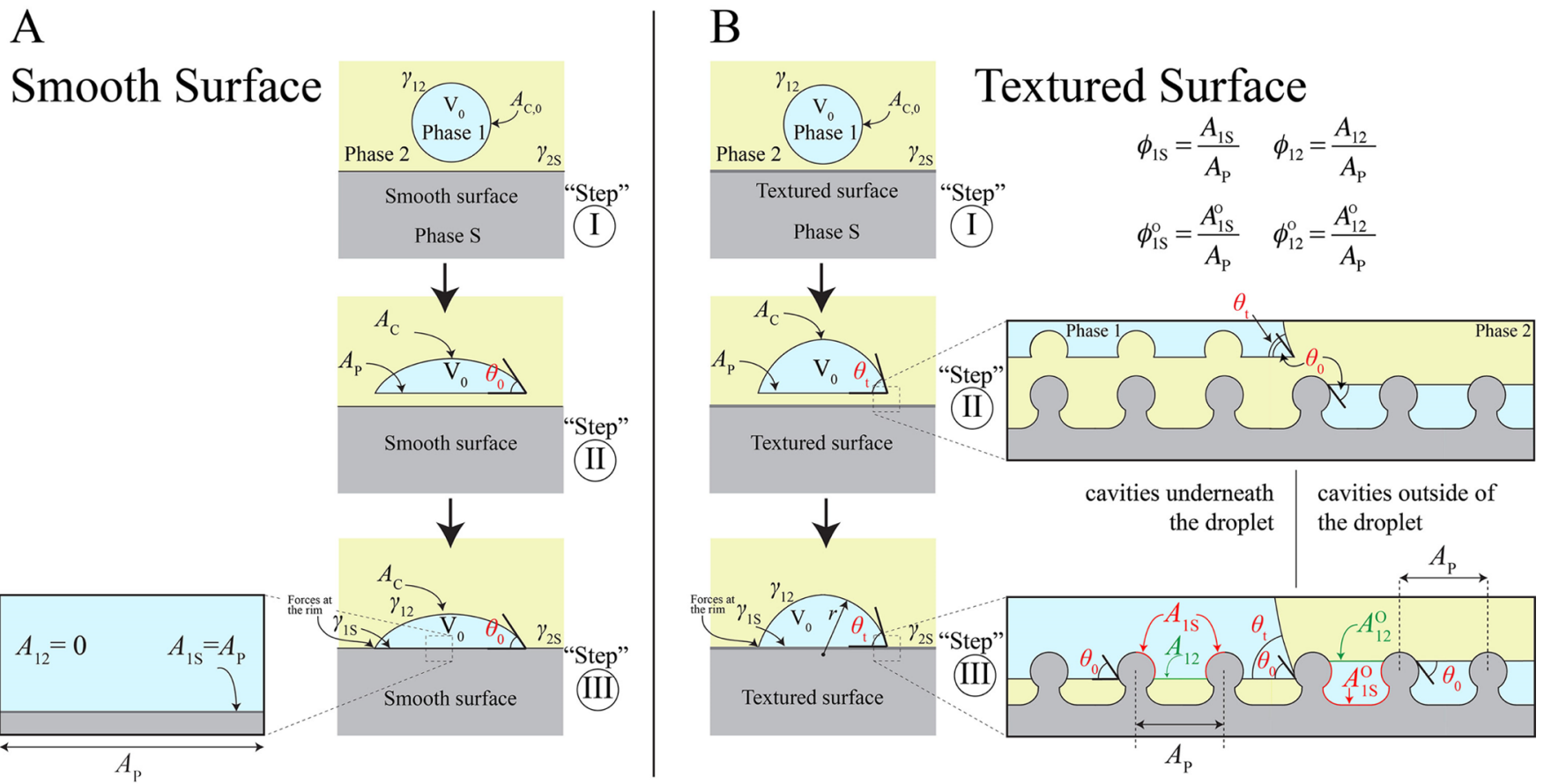

Figure 2. Illustration of the energy minimization procedure used to derive eqs 8 (Young equation) and 10 ("wetting equation"). The same energy minimization procedure has been used by others. ${ }^{16,48}$ Equation 10 is a "wetting equation" that can be used to calculate the contact angle on smooth and textured (re-entrant or not) surfaces for the four different wetting states as defined in ref 37 and Figure 3. Equation 8 was derived as follows: A spherical Phase 1 drop in the presence of Phase 2 (Step I) is deformed into a spherical cap with curved and flat areas (Step II), and thus the surface energy increases. As the spherical cap comes in contact with the smooth solid, the surface energy decreases and reaches thermodynamic equilibrium (Step III). The same approach was employed for the deriving the analogous equation for use with textured surfaces, eq 10; notably, in the latter derivation, the interfacial energy contributions from underneath and outside of the droplet are due to combinations of Phase $1-\mathrm{Phase} 2\left(A_{12}\right)$, Phase 1 -Solid $\left(A_{1 \mathrm{~S}}\right)$, and Phase 2-Solid $\left(A_{2 \mathrm{~S}}\right)$ interfaces.

wetting states exhibit significantly different contact angles (the difference can be up to $\sim 180^{\circ}$ ); hence, from an engineering point of view, it would be advantageous to be able to design textures that would promote a certain wetting state. For example, for the preparation of superhydrophilic surfaces $\left(\theta_{t} \sim\right.$ $0^{\circ}$ ), the "fully filled I empty" wetting state (Wenzel state) is favorable when $\theta_{0}<90^{\circ}$ (illustrated in Figure 1B). According to the Wenzel equation, discussed below, the contact angle in the "fully filled I empty" state, $\theta_{\mathrm{t}}^{\mathrm{fe}}$, is smaller than $\theta_{0}$ when $\theta_{0}<90^{\circ}$, whereas for omniphobic surfaces $\left(\theta_{\mathrm{t}}>90^{\circ}\right.$, contact angles for water and oils), the "partially filled I empty" state (CassieBaxter state) should be favored (see Figure 1C). In the extreme case, more than $90 \%$ of the liquid that is resting on the surface is in contact with the vapor rather than the solid surface (Figure 1C). Therefore, from an energy point of view, there is no significant difference between a drop of liquid purely in contact with vapor (i.e., drop that is not in contact with a solid) and a drop that is placed on a textured omniphobic surface. Thus, surfaces can be engineered such that $\theta_{\mathrm{t}}^{\mathrm{pe}}$ can approach $\sim 160^{\circ}$ or higher for any liquid. ${ }^{21}$

Recently, ${ }^{2,21}$ it has been shown that re-entrant and doubly reentrant structures-cavities that broaden below the surfaceare able to stabilize the "partially filled I empty" wetting state. However, as mentioned above, using the current theoretical models, ${ }^{2,35,40,41}$ intensive mathematical manipulations are required to predict a priori: (1) which wetting state ("fully filled I empty", "partially filled I empty", "partially filled | partially filled", or "fully filled I partially filled") would result for a given surface texture and $\theta_{0}$, and (2) whether that wetting state is transient, metastable, or thermodynamically stable.
In this paper, we consider a sessile drop that forms a truncated sphere of $<500 \mu \mathrm{m}$ base radius, $R_{\mathrm{b}}$ (Figure 1C), on an infinitely large (in comparison to $R_{\mathrm{b}}$ ) nondeformable array of axisymmetric cavities/protrusions, which represents a commonly studied complex system. The situation of randomly rough profiles is entirely analyzable, but only once this profile is known. Unfortunately, it is not possible to have a unique equation that applies to randomly rough profiles because these have an infinite number of possible configurations, each of which will produce a different result. Based on the size of the droplet, we assume that the effects of line tension can be neglected. ${ }^{42,43}$ We also assume that the diameter of a single cavity is significantly smaller than the capillary length (defined in the derivation of the "Local Force Equation" section below); therefore surface tension, not gravitational forces, dominates the static and dynamic properties of the system. Lastly, our model-as presented here-does not predict the effects of contact angle hysteresis; however, the model can be extended to address these effects, as discussed in the Experimental section.

Based on the aforementioned assumptions, we present a wetting model that can be used to predict the metastable and thermodynamically stable states and contact angles on any surface with nondeformable axisymmetric (symmetric around the depth axis of the cavity/protrusion) array of micro/ nanotextures on the metastable and thermodynamically stable contact angles. The equations we derived can be easily applied on any axisymmetric array of fabricated or natural cavities/ protrusions, as demonstrated on a complex but not atypical cavity geometry in the Experimental section. 
The article starts with a Theory section, where we present the derivation of the "wetting equation". The "wetting equation" predicts the contact angle on any textured/ patterned/rough surface, $\theta_{\mathrm{t}}$. The "wetting equation" combines the Wenzel and Cassie-Baxter equations into a single unified equation and also considers the effects of the partially filled or empty cavities outside the droplet (also called the "impregnated wetting state" $\left.{ }^{18,44-46}\right)$ on $\theta_{\mathrm{t}}$. We then introduce a new local "energy minimum" or "force balance" equation - an equation that indicates whether a cavity will get partially or fully filled by an intruding liquid depending on its geometry. Next, we introduce a "total interfacial energy analysis" to determine whether the cavities both under and outside the droplet will get partially or fully filled when thermodynamic equilibrium is achieved. We end the paper with an Experimental section, in which we demonstrate how our wetting model can be applied for predicting/engineering the metastable and the thermodynamic contact angles on premicrofabricated re-entrant and nonre-entrant cavities. In the experimental demonstration, we also discuss the limitations of the current model to describe contact angle hysteresis.

\section{THEORY}

2.1. Derivation of a "Wetting Equation". In this section a "wetting equation" is derived that can be used to calculate the contact angle on textured surfaces, $\theta_{\mathrm{t}}$, for any given wetting state. We show that the "wetting equation", under certain conditions, reduces to the well-known Cassie-Baxter, Wenzel, and Young equations. More importantly, the "wetting equation" reveals that the state of the cavities/pores outside the droplet-whether they are empty or partially filled-affects the contact angle, which is often not considered, even though it has been discussed before. ${ }^{47}$

To elucidate our methodology of derivation, we start with a flat and smooth surface (Figure 2A). The contact angle is described by the Young and Young-Dupré equations, where for a droplet of Phase 1 on a solid surface $S$ in the presence of Phase 2 these may be written as

Young equation:

$$
\gamma_{12} \cos \theta_{0}=\gamma_{2 S}-\gamma_{1 S}
$$

Young-Dupré equation:

$$
\gamma_{12}\left(1+\cos \theta_{0}\right)=W_{0}
$$

where $\gamma_{12}, \gamma_{1 S}$, and $\gamma_{2 S}$ are the surface/interfacial tensions of the Phase 1-Phase 2, Phase 1-Solid, and Phase 2-Solid interfaces, respectively, and $W_{0}$ is the adhesion energy of bringing unit area of Phase 1 and solid into contact in the presence of Phase 2. Traditionally, the Young equation is derived by balancing the forces due to interfacial tensions in the horizontal plane. However, from a thermodynamic perspective, it is more appropriate to derive these equations by minimizing the total interfacial energy of the system (and this approach should anyway give the same result as the force balance approach). ${ }^{41,48}$

The energy minimization approach can be split into three steps. In the first step, a reference step (i.e., surface energy $E=$ 0 , see Figure 2A, Step I), we assume a spherical drop of surface area, $A_{\mathrm{C}, 0}$. We also assume that the volume of the drop, $V_{0}$, does not change with time and that the drop diameter is significantly smaller than the capillary length, $\sqrt{\frac{\gamma_{12}}{\rho g}}$, where $g$ is the gravitational acceleration and $\rho$ the mass density of the drop.
Therefore, gravitational forces are significantly smaller than the surface forces. In the second step, the spherical drop deforms to a truncated spherical structure and exhibits curved area, $A_{\mathrm{C}}$, and projected, flat area, $A_{\mathrm{p}}$. The region encompassed by this projected area is subsequently referred to as the "area under the wetting droplet", whereas the region beyond the three-phase contact line of the droplet and the solid surface is referred to as the "area outside the wetting droplet" (see Figure 2A, Step II). Note that the angle between $A_{\mathrm{C}}$ and $A_{\mathrm{P}}$ is the equilibrium contact angle, $\theta_{0}$, and the change in energy, in comparison to Step I, is $\Delta E_{\mathrm{I} \rightarrow \mathrm{II}}=\gamma_{12}\left(A_{\mathrm{C}}-A_{\mathrm{C}, 0}\right)+\gamma_{12} A_{\mathrm{P}}$. In the third step, the flat area of the liquid, $A_{\mathrm{p}}$, comes into contact with the solid; hence the surface energies, $\gamma_{12} A_{\mathrm{P}}$ and $\gamma_{2 \mathrm{~S}} A_{\mathrm{P}}$, are lost, and the total interfacial surface energy, $E_{0}$, (using Step I as a reference) is given by

$$
E_{0}=\gamma_{12}\left(A_{\mathrm{C}}-A_{\mathrm{C}, 0}\right)+\left(\gamma_{1 \mathrm{~S}}-\gamma_{2 \mathrm{~S}}\right) A_{\mathrm{P}}
$$

In a subsequent step, which is not shown in Figure 2A, if an infinitesimally small perturbation is made from the equilibrium configuration, which involves an increase or decrease in Phase 1 -Phase 2 and Phase $1-$ Solid interfaces by $\mathrm{d} A_{\mathrm{C}}$ and $\mathrm{d} A_{\mathrm{P}}$, the interfacial energy changes to

$$
E_{0}+\mathrm{d} E=\gamma_{12}\left(A_{\mathrm{C}}+\mathrm{d} A_{\mathrm{C}}-A_{\mathrm{C}, 0}\right)+\left(\gamma_{1 \mathrm{~S}}-\gamma_{2 \mathrm{~S}}\right)\left(A_{\mathrm{P}}+\mathrm{d} A_{\mathrm{P}}\right)
$$

The contact angle changes to $\theta_{0}+\mathrm{d} \theta$, and the change in the interfacial energy is given by

$$
\mathrm{d} E=\gamma_{12}\left(\mathrm{~d} A_{\mathrm{C}}\right)+\left(\gamma_{1 \mathrm{~S}}-\gamma_{2 \mathrm{~S}}\right)\left(\mathrm{d} A_{\mathrm{P}}\right)
$$

Since the Phase 1 drop was at thermodynamic equilibrium with total interfacial surface energy $E_{0}$, the derivative, $\mathrm{d} E$, with respect to $\theta, A_{\mathrm{C}}$, and $A_{\mathrm{P}}$ at constant droplet volume should be zero. Setting this condition and rearranging eq 5 , we get

$$
\left(\frac{\mathrm{d} E}{\mathrm{~d} A_{\mathrm{P}}}\right)_{V_{0}}=\gamma_{12}\left(\frac{\mathrm{d} A_{\mathrm{C}}}{\mathrm{d} A_{\mathrm{P}}}\right)_{\mathrm{V}_{0}}+\left(\gamma_{1 \mathrm{~S}}-\gamma_{2 \mathrm{~S}}\right)=0
$$

Next, we employ a geometric relationship that holds true for all spherical sections of constant volume $V_{0}$, contact angle $\beta$, and curved and flat areas, $A_{\mathrm{C}}$ and $A_{\mathrm{P}}$, respectively (derivation presented in the Supporting Information Section 2)

$$
\left(\frac{\mathrm{d} A_{\mathrm{C}}}{\mathrm{d} A_{\mathrm{P}}}\right)_{V_{0}}=\cos \beta
$$

Inserting eq 7 into eq 6 and substituting $\beta=\theta_{0}+\mathrm{d} \theta$ we get

$$
\left(\frac{\mathrm{d} E}{\mathrm{~d} A_{\mathrm{P}}}\right)_{V_{0}}=\gamma_{12} \cos \left(\theta_{0}+\mathrm{d} \theta\right)+\left(\gamma_{1 \mathrm{~S}}-\gamma_{2 \mathrm{~S}}\right)=0
$$

which yields the Young equation

$$
\gamma_{12} \cos \theta_{0}=\gamma_{2 S}-\gamma_{1 S}
$$

For completeness, we take the second derivative of energy with respect to $A_{\mathrm{P}}$ and find

$$
\left(\frac{d^{2} E}{d A_{\mathrm{P}}^{2}}\right)_{V_{0}}=\frac{\mathrm{d} \theta}{\mathrm{d} A_{\mathrm{P}}} \frac{\mathrm{d}}{\mathrm{d} \theta}\left(\frac{\mathrm{d} E}{\mathrm{~d} A_{\mathrm{P}}}\right)=\frac{\gamma_{12}(2+\cos \theta)}{2 \pi R^{2}}>0
$$

which is true for all $\theta$ and, hence, proves that it is an energy minimum (not necessarily the global minimum).

Using the above approach, a "wetting equation" was derived, which includes the effects of surface texture. (The full 

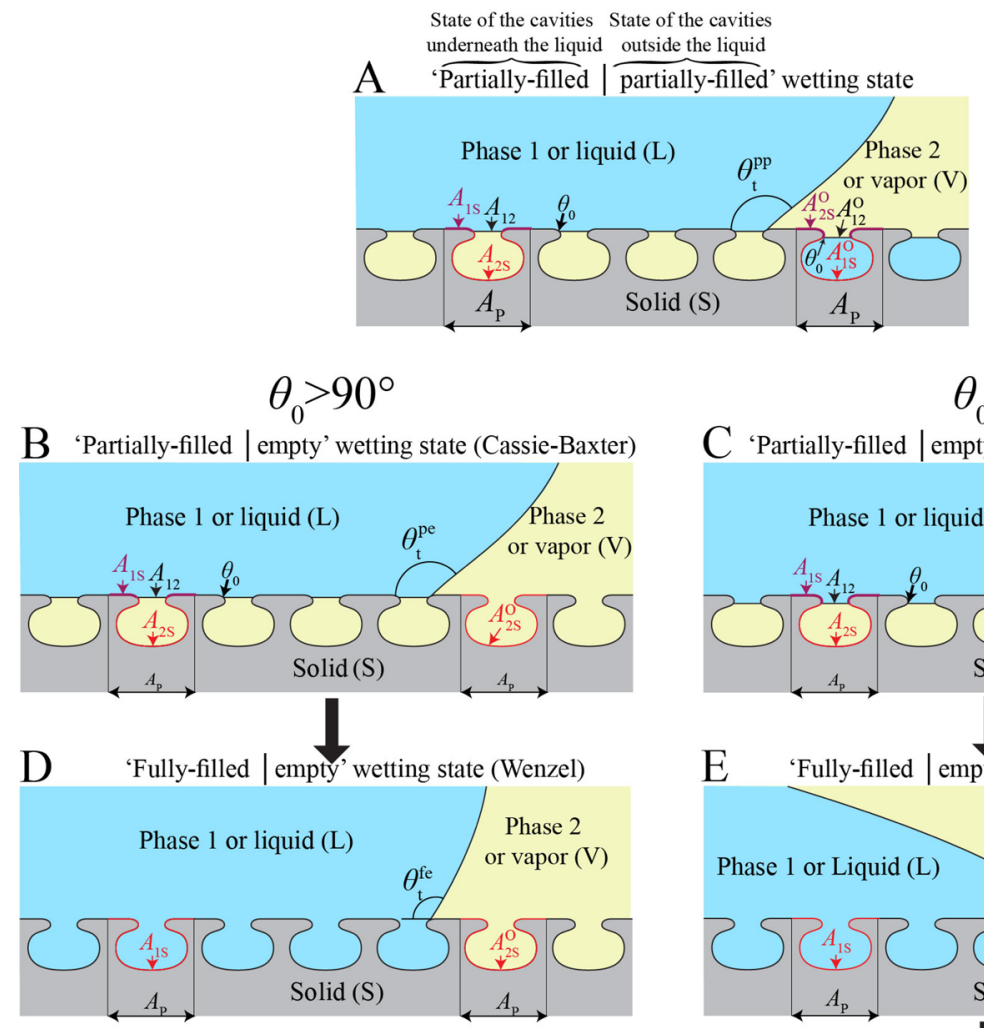

Contact-area between phases $\mathrm{X}$ and $\mathrm{Y}$ und
where $\mathrm{X}$ and $\mathrm{Y}$ can be either Phase 1, Phase
Solid $\mathrm{S}$
$\phi_{\mathrm{XY}}=\frac{A_{\mathrm{XY}}}{A_{\mathrm{P}}}, \quad \phi_{\mathrm{XY}}^{\mathrm{O}}=\frac{A_{\mathrm{XY}}}{A_{\mathrm{P}}}$,
Projected area of a repeating texture unit

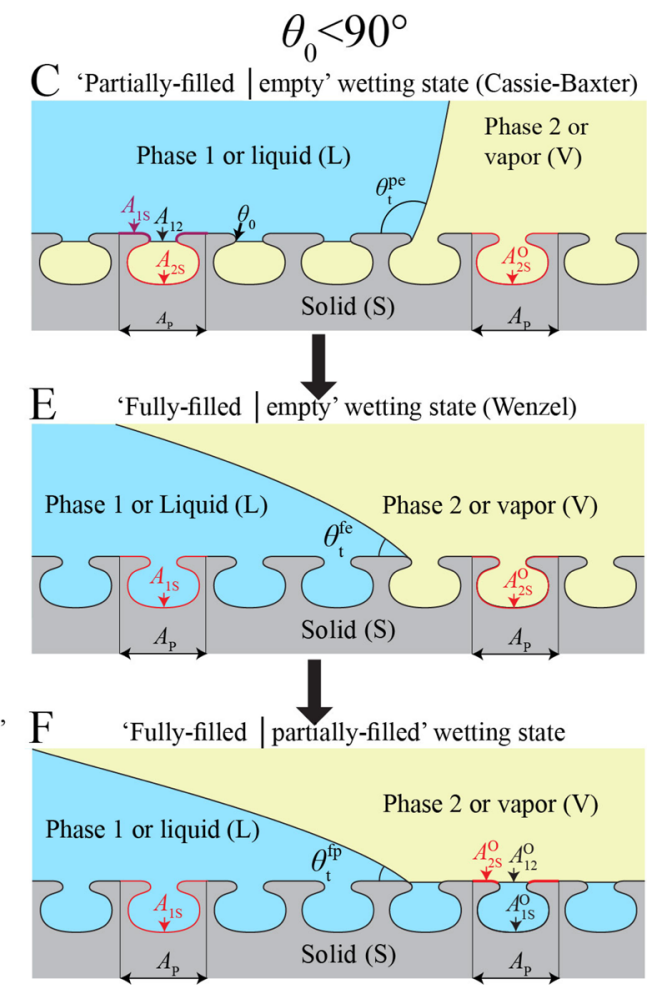

Figure 3. Schematic illustration of the four possible extreme wetting states, as defined in ref 37 . In practice, the wetting state is a combination of these four wetting states. The "wetting equation", eq 10, predicts the contact angle for the "partially filled I partially filled" wetting state depicted in Panel A. The "wetting equation" can then be reduced to the "partially filled I empty" wetting state (Cassie-Baxter), as shown in Panels B and C, or the "fully filled I empty" wetting state (Wenzel) as shown in Panels D and E. In addition, as illustrated in Panel F, the "wetting equation" can be reduced to a "fully filled I partially filled" wetting state. Note that a cavity is considered fully filled if there is no Phase 1-Phase 2 interface (or no liquid-vapor interface) anywhere within the cavity. Note also that the cavities can be connected or disconnected to adjacent cavities.

derivation can be found in the Supporting Information Section 3)

$$
\cos \theta_{\mathrm{t}}=\cos \theta_{0}\left(\phi_{1 \mathrm{~S}}-\phi_{1 \mathrm{~S}}^{\mathrm{O}}\right)-\left(\phi_{12}-\phi_{12}^{\mathrm{O}}\right)
$$

where $\theta_{\mathrm{t}}$ is the apparent contact angle on the textured surface, $\phi_{1 \mathrm{~S}}=A_{1 \mathrm{~S}} / A_{\mathrm{p}}, \phi_{1 \mathrm{~S}}^{\mathrm{O}}=A_{1 \mathrm{~S}}^{\mathrm{O}} / A_{\mathrm{p}}, \phi_{12}=A_{12} / \mathrm{A}_{\mathrm{p}}$, and $\phi_{12}^{\mathrm{O}}=A_{12}^{\mathrm{O}} / \mathrm{A}_{\mathrm{p}}$; $A_{1 S}$ and $A_{12}$ (Figure $2 \mathrm{~B}$ ) are the interfacial areas between Phase 1 and the Solid and between Phase 1 and Phase 2 under the drop, respectively. $A_{1 \mathrm{~S}}^{\mathrm{O}}$ and $A_{12}^{\mathrm{O}}$ are the interfacial areas between Phase 1 and the Solid and between Phase 1 and Phase 2 outside the drop, respectively. $A_{\mathrm{P}}$ is the projected area of the repeating texture ("unit cell"). We note that eq 10 corresponds to the "partially filled I partially filled" wetting state, which can only be metastable as elaborated below.

As mentioned above, under certain conditions, the "wetting equation", eq 10, reduces to the Cassie-Baxter, Wenzel, "impregnated", and Young equations. In order to get the Cassie-Baxter equation, which is the "partially filled I empty" wetting state (see Figures $3 \mathrm{~A}$ and $\mathrm{B}$ ), we note that all the cavities outside the droplet are assumed to be of Phase 1 (or filled with Phase 2) (i.e., $\phi_{1 \mathrm{~S}}^{\mathrm{O}}=\phi_{12}^{\mathrm{O}}=0$ ) and that in the final state the solid surface is wetted by Phase 1 and cavities under the droplet are partially filled with Phase 1 from the top of the cavities (i.e., $\phi_{1 S}>0$ and $\phi_{12} \geq 0$ ). Under these conditions eq 10 reduces to

$$
\cos \theta_{\mathrm{t}}^{\mathrm{pe}}=\phi_{1 \mathrm{~S}} \cos \theta_{0}-\phi_{12}
$$

which is the general form of the Cassie-Baxter model for a Phase 1-Phase 2-Solid system. When Phase 1 is Liquid and Phase 2 is Vapor, replacing $1 \rightarrow \mathrm{L}$ and $2 \rightarrow \mathrm{V}$, we obtain the original Cassie-Baxter equation

$$
\cos \theta_{\mathrm{t}}^{\mathrm{pe}}=\phi_{\mathrm{LS}} \cos \theta_{0}-\phi_{\mathrm{LV}}
$$

where $\phi_{\mathrm{LS}}+\phi_{\mathrm{LV}} \geq 1$. Note that it is a common mistake to assume that $\phi_{\mathrm{LS}}+\phi_{\mathrm{LV}}=1$ is true for all textures.

Similarly, the Wenzel equation is obtained for the case where the cavities under the droplet become fully filled with Phase 1, i.e. $\frac{A_{12}}{A_{\mathrm{p}}}=\frac{A_{2 S}}{A_{\mathrm{p}}}=0 \Rightarrow \phi_{12}=\phi_{2 \mathrm{~S}}=0$, and the cavities outside the droplet remain empty of Phase 1 (i.e., $\phi_{1 S}^{\mathrm{O}}=\phi_{12}^{\mathrm{O}}=0$ ). This 


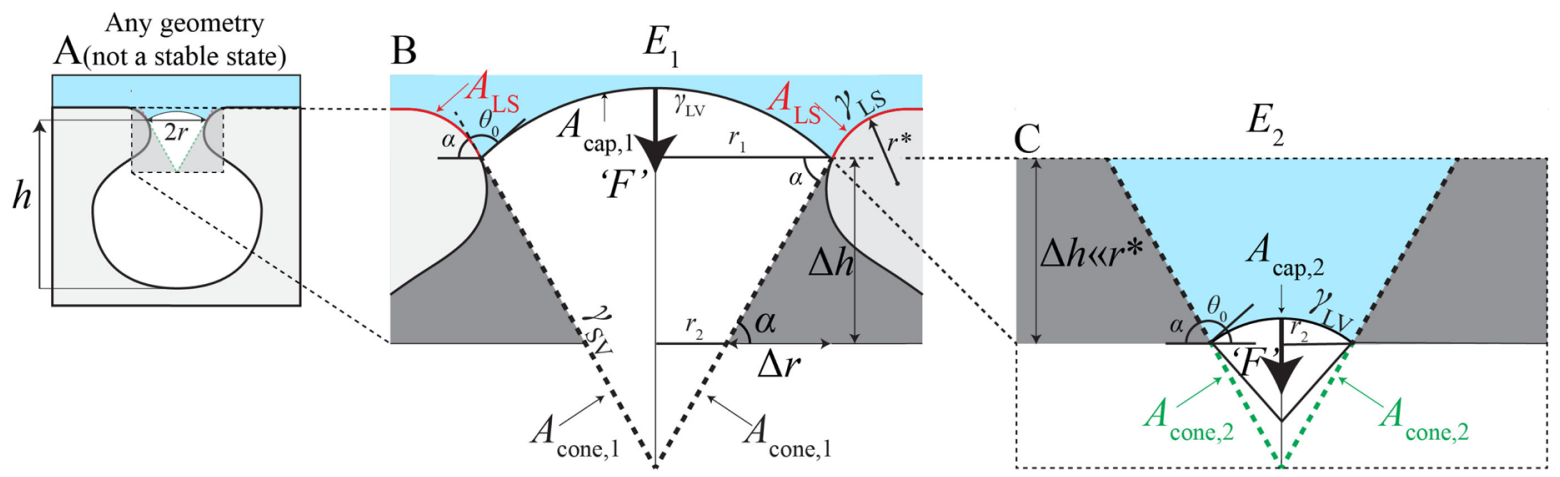

Figure 4. For any axisymmetric cavity one can draw an imaginary cone that is locally tangent to the cavity, as depicted in Panel A. The "local force equation" was derived based on the change in local interfacial surface energies of the partially and fully filled cone, as depicted in Panels B and C, respectively. The result is a "local force equation" that can be used to calculate the force (magnitude and direction), " $F$ ", defined by $F=-d E / d h$, that acts on the liquid-vapor interface in a cavity. " $F$ " can either force or "push" the liquid into (" $F$ " $<0$ ) or "pull it" out of (" $F$ " $>0)$ the cavity. In the absence of inertial forces, it is found that a planar, i.e., flat liquid-vapor, interface is stable (neither penetrating nor ejecting out of the cavity) when the local energy is minimum and " $F "=0$. We note that this is also the condition where the Laplace pressure across the liquid-vapor interface is zero.

is the "fully filled I empty" wetting state (see Figures $3 \mathrm{C}$ and $3 \mathrm{D})$, and under these conditions, eq 10 reduces to

$$
\cos \theta_{\mathrm{t}}^{\mathrm{fe}}=\phi_{1 \mathrm{~S}} \cos \theta_{0}
$$

which is the general form of the Wenzel equation for liquidliquid-solid systems. Again, if Phase 1 is Liquid and Phase 2 is Vapor, then eq 13 reduces to the original Wenzel equation ${ }^{30}$

$$
\cos \theta_{\mathrm{t}}^{\mathrm{fe}}=\phi_{\mathrm{LS}} \cos \theta_{0}=r \cos \theta_{0}
$$

where $r(\geq 1)$ is the "roughness" parameter in the original Wenzel equation, which is the ratio between the real 1-S (LS) contact area, $A_{1 S}$, to the projected area, $A_{\mathrm{P}}$.

Both the Cassie-Baxter and Wenzel models implicitly assume that the cavities outside the droplet remain empty; however, when $\theta_{0}<90^{\circ}$, as elaborated below, these cavities can get filled due to condensation or drainage of Phase 1 into the cavities, and the "fully filled I partially filled" wetting state can be established (see Figure 3F). As with the "fully filled I empty" scenario, $\phi_{12}=0$. However, in this case, the cavities outside of the droplet are partially filled with Phase 1 , thus $\phi_{1 \mathrm{~S}}^{\mathrm{O}}>0$ and $\phi_{12}^{\mathrm{O}}$ $\geq 0$. Under these conditions, eq 10 reduces to

$$
\cos \theta_{\mathrm{t}}^{\mathrm{fp}}=\cos \theta_{0}\left(\phi_{1 \mathrm{~S}}-\phi_{1 \mathrm{~S}}^{\mathrm{O}}\right)+\phi_{12}^{\mathrm{O}}
$$

which can be further simplified, based on geometric relations (see Figure 3F), to

$$
\cos \theta_{\mathrm{t}}^{\mathrm{fp}}=\phi_{2 \mathrm{~S}}^{\mathrm{O}} \cos \theta_{0}+\phi_{12}^{\mathrm{O}}
$$

When Phase 1 is Liquid and Phase 2 is Vapor, eq 16 becomes

$$
\cos \theta_{\mathrm{t}}^{\mathrm{fp}}=\phi_{\mathrm{SV}}^{\mathrm{O}} \cos \theta_{0}+\phi_{\mathrm{LV}}^{\mathrm{O}}
$$

Lastly, when the surface is smooth, $\phi_{1 \mathrm{~S}}=1$ and $\phi_{1 \mathrm{~S}}^{\mathrm{O}}=\phi_{12}=\phi_{12}^{\mathrm{O}}$ $=0$, and eq 10 reduces to

$$
\cos \theta_{\mathrm{t}}=\cos \theta_{0}
$$

The contact angle in eq 18 is given by the Young equation.

To summarize this section, eq 10 is a "wetting equation" that can be used to calculate $\theta_{\mathrm{t}}$ of a Phase 1-Phase 2-Solid system, which can be used for the four different wetting states. In the next sections, we derive and demonstrate a new method that can be used to calculate the different area fractions, viz., $\phi_{1 S}$, $\phi_{12}, \phi_{1 S}^{\mathrm{O}}$, and $\phi_{12}^{\mathrm{O}}$.
2.2. Local Force (and Energy) Equation. To determine a priori the different area fractions $-\phi_{\mathrm{LS}}, \phi_{\mathrm{LV}}, \phi_{\mathrm{LV}}^{\mathrm{O}}$, and $\phi_{\mathrm{LS}}^{\mathrm{O}}-$ which are required for calculating the contact angles using the "wetting equation" (eq 10), one needs to determine whether the liquid penetrates into the cavity/pores or not. To answer this question, a "local force equation" is derived using a similar approach that was proposed by Tsori. ${ }^{49,50}$ The "local force equation" determines the direction of the local force, " $F$ ", defined by $F=-d E / d h$, at the liquid-vapor interface in the cavity. More importantly, the "local force equation" determines whether the energy decreases (favorable) or increases (not favorable) as the liquid-vapor interface penetrates the cavity, i.e., varies with cavity height, $h$. As we demonstrate below, " $F$ " can force the liquid-vapor interface into or out of the cavity. In addition, the "local force equation" determines whether condensation in a cavity is energetically favorable or not.

For the derivation of the "local force equation", let us assume that the largest radius of the cavity, $r$ (Figure 4A), is significantly smaller than the capillary length; hence gravitational forces are negligible in comparison to the surface tension forces $\left(r \ll \sqrt{\frac{\gamma_{\mathrm{LV}}}{\rho g}}\right.$ or Bond Number, $B_{0}<0.1$, where $\rho$ is the mass density of the liquid and $g$ is the gravitational constant). Also, for the sake of simplicity, a Solid (S)-Liquid (L)-Vapor (V) system is considered. Lastly, we assume an axisymmetric cavity about the vertical axis, $h$ (Figure 4 ).

As shown in Figure 4A, at any given height, $h$, in the cavity one can draw an imaginary cone with a slope of $\alpha$ that is tangent to the cavity. We note that the imaginary cone implies that for any axisymmetric cavity $\alpha$ is constant for $\Delta h \rightarrow 0 .{ }^{51}$ The virtual cone shape is chosen for convenience of the following calculation and not a characteristic of the real (e.g., re-entrant cavity) surface. When a liquid comes into contact with this cone-shaped cavity, the intrinsic contact angle, $\theta_{0}$, is formed along the liquid-solid-vapor rim (see Figure 4B), which curves the liquid-vapor interface. The surface energy of this partially filled cone is (Figure 4B)

$$
E_{1}=A_{\text {cap }, 1} \gamma_{\mathrm{LV}}+A_{\text {cone }, 1} \gamma_{\mathrm{SV}}+A_{\mathrm{LS}} \gamma_{\mathrm{LS}}
$$

where $A_{\text {cone, } 1}$ is the area of the cone that is in contact with vapor, as shown in Figure 4B; $A_{\text {cap }, 1}$ is the area of the liquidvapor interface; and $A_{\mathrm{LS}}$ is the area of the cone that is in contact with the liquid in Figures $4 \mathrm{~B}$ and $\mathrm{C}$. 

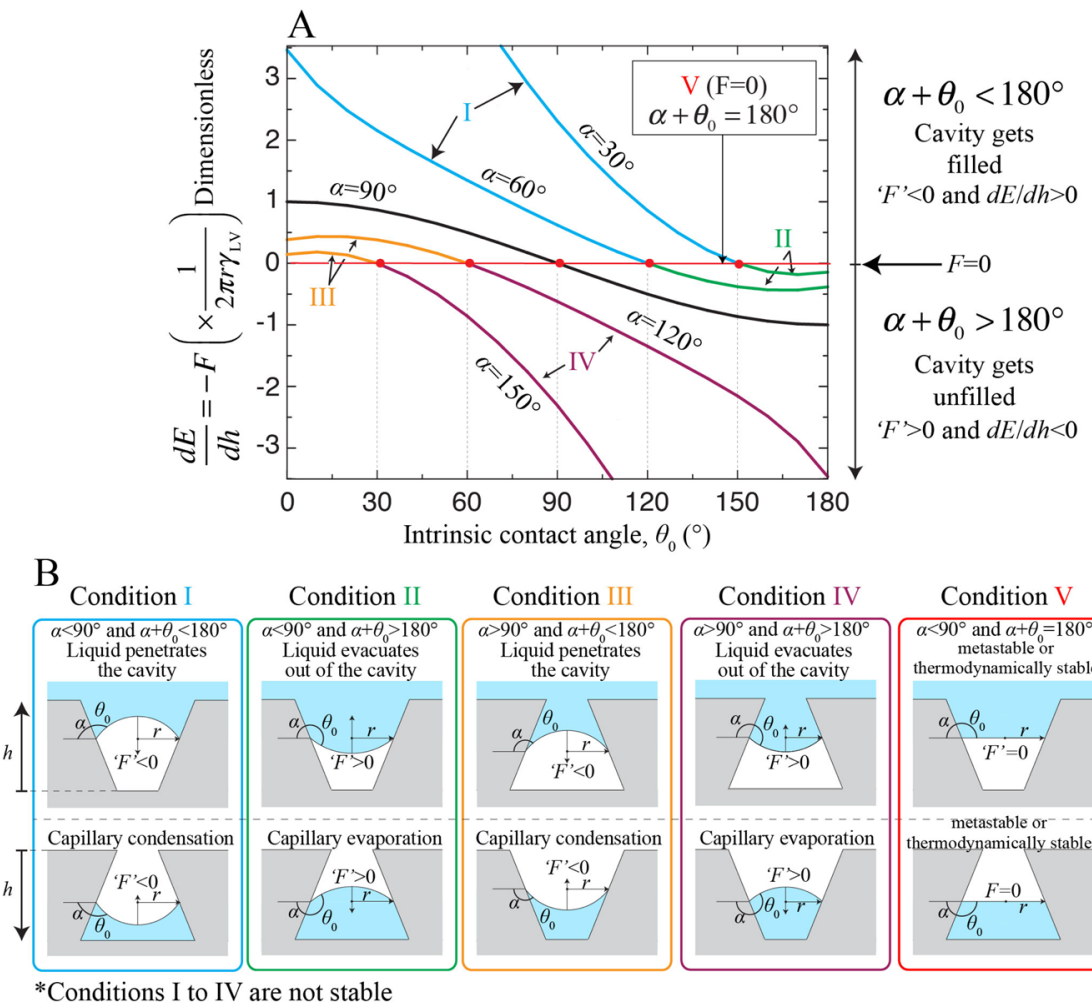

Condition $\mathrm{V}$

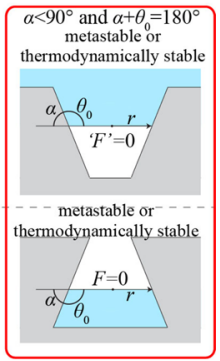

Conditions I to IV are not stable

Figure 5. Panel A is a plot of the "local force equation" ( $y$-axis shows the sum of the terms in the brackets in eq 21), showing that when $\alpha+\theta_{0}<$ $180^{\circ}$, then $F<0$. The liquid is pushed into the cavity, and "capillary condensation" takes place; i.e., liquid condenses even if the vapor is not supersaturated. The case where $F<0$ is schematically depicted in Panel B, Conditions I and III. If $\alpha+\theta_{0}>180^{\circ}$, then $F>0$. Liquid is pushed out of the cavity, and "capillary evaporation" is favorable; i.e., evaporation is favorable even in saturated vapor. This case is depicted in Panel B, Conditions II and IV. When $\alpha+\theta_{0}=180^{\circ}$, as depicted in Panel B, Condition V, the liquid is stable (not moving) in the absence of external forces.

When the liquid penetrates the cone by $\Delta h$ and $\Delta h$ is significantly smaller than the surface curvature, $r^{*}$ (Figure 4C), the surface energy is given by

$$
E_{2}=A_{\text {cap }, 2} \gamma_{\mathrm{LV}}+A_{\text {cone }, 2} \gamma_{\mathrm{SV}}+A_{\mathrm{LS}} \gamma_{\mathrm{LS}}+\left(A_{\text {cone }, 1}-A_{\text {cone }, 2}\right) \gamma_{\mathrm{LS}}
$$

where $A_{\text {cap } 22}$ and $A_{\text {cone, } 2}$ are the areas of the liquid-vapor and the solid-vapor interfaces, respectively, as shown in Figure 4C.

Assuming that the liquid-vapor interface in Figure 4B is a truncated sphere, thus

$$
A_{\text {cap }, 1}=\frac{2 \pi r_{1}^{2}}{1-\cos \left(\alpha+\theta_{0}\right)}, A_{\text {cap }, 2}=\frac{2 \pi r_{2}^{2}}{1-\cos \left(\alpha+\theta_{0}\right)}
$$

and

$$
\begin{aligned}
& A_{\text {cone }, 1}=\frac{\pi r_{1}^{2}}{\cos \alpha}, A_{\text {cone }, 2}=\frac{\pi r_{2}^{2}}{\cos \alpha}, \\
& \quad \gamma_{\mathrm{LV}} \cos \theta_{0}=\gamma_{\mathrm{SV}}-\gamma_{\mathrm{LS}}, r_{1}=r_{2}+\frac{\Delta h}{\tan \alpha}
\end{aligned}
$$

where $r_{1}$ and $r_{2}$ are the two radii of the liquid-solid-vapor rim for two different penetration depths of the liquid (Figures $4 \mathrm{~B}$ and $\mathrm{C}$ ), and the infinitesimal change in interfacial energy as the liquid penetrates the cone is given by

$$
\begin{aligned}
& -\left.\frac{\Delta E}{\Delta h}\right|_{\Delta h \rightarrow 0}=-\frac{d E}{d h}={ }^{\prime} F^{\prime}=-2 \pi r \gamma_{L V}\left[\frac{2}{\left[1-\cos \left(\alpha+\theta_{0}\right)\right] \tan \alpha}\right. \\
& \left.+\frac{\cos \theta_{0}}{\sin \alpha}\right]
\end{aligned}
$$

where $r$ is the cavity opening radius at a given $h$ (Figure 4A).
Throughout this paper we refer to eq 21 as the "local force equation". We note that in eq $21 \mathrm{dE} / \mathrm{dh}$ has units of force, which is the total force acting nonuniformly on the liquidvapor interface and normal to the surface at the liquid-solidvapor rim. ${ }^{52}$ Here, we focus on the change in local interfacial energy in a single cavity, which ultimately determines whether liquid penetrates, remains stationary, or evacuates from the cavity (the total interfacial energy of the system is considered in Section 2.4). By considering the change in energy, as opposed to force balances, we avoid the unnecessary complication of specifying exactly at which point or points this "force" acts.

Figure 5A shows a plot of the normalized force $\left(-\frac{F}{2 \pi r \gamma_{\mathrm{LV}}}\right)$ versus the intrinsic contact angle, $\theta_{0}$, for different values of $\alpha$ using the "local force equation", eq 21. The plot shows that (1) for $\alpha+\theta_{0}<180^{\circ}$, where the liquid-vapor interface is concave (see Figure 5B upper row, Conditions I and III), the interfacial energy increases with $h\left(\frac{d E}{d h}>0\right)$, and " $F$ " $(<0)$ forces the liquid to penetrate into the cavity, which also promotes liquid condensation (see Figure 5B lower row, Conditions I and III). Note that $h$ and $\alpha$ are inverted in the case of penetration/ ejection (upper row of Figure 5B) in comparison to the case of condensation/evaporation of the liquid in the cavity (lower row of Figure 5B). (2) For $\alpha+\theta_{0}>180^{\circ}$, where the liquid-vapor interface is convex (see Figure 5B Conditions II and IV), the energy decreases with $h\left(\frac{d E}{d h}<0\right)$, and " $F$ " $>0$; thus " $F$ " forces the liquid out of the cavity, and condensation is prevented (or evaporation is promoted). (3) For $\alpha+\theta_{0}=180^{\circ}$ (see Figure 5B Condition V), the liquid-vapor interface is flat, and the 


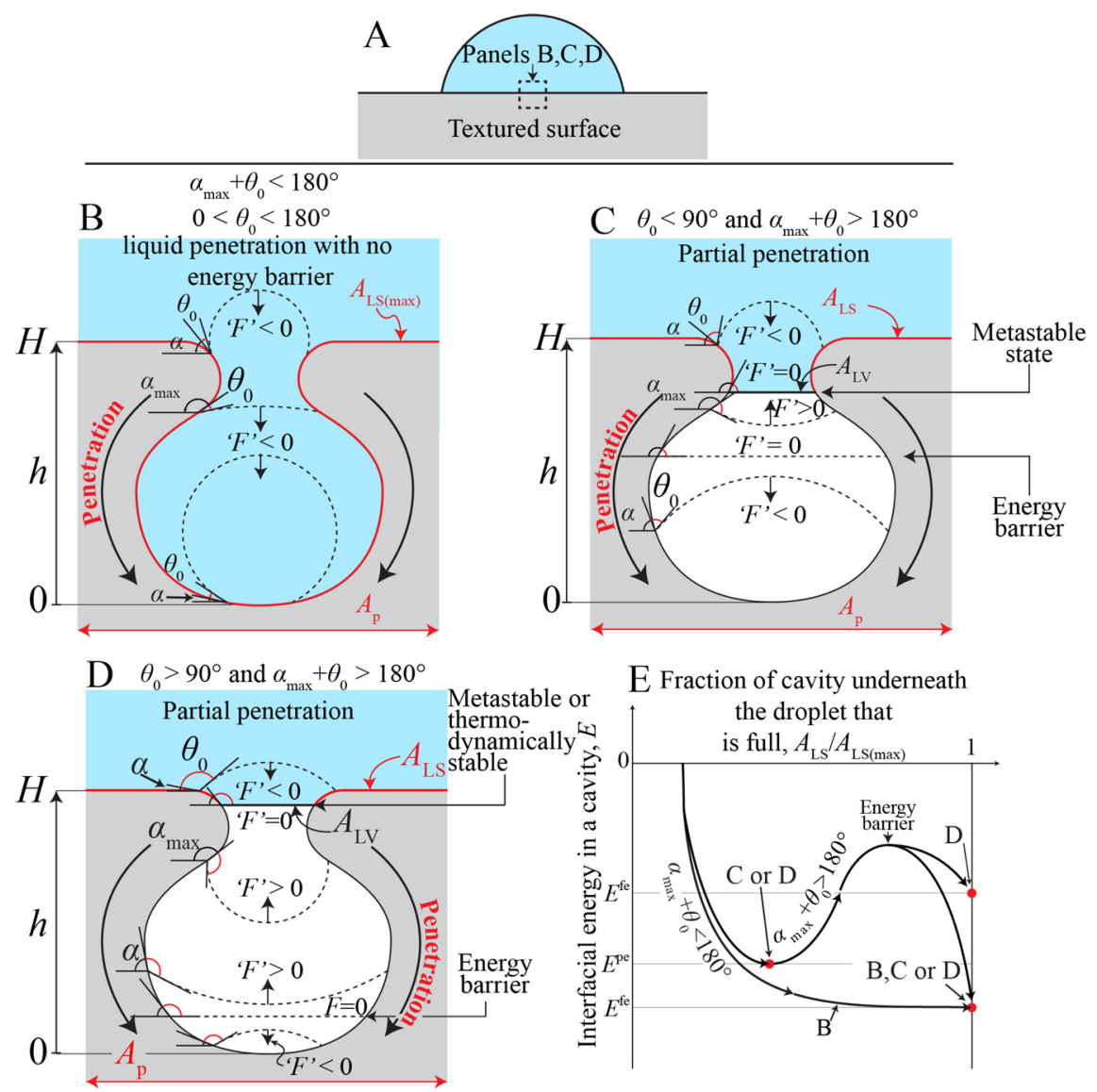

Figure 6. Schematic illustration of the cavities under the droplet (Panel A) and the effect of the maximum slope, $\alpha_{\max }$. In Panel B, when $\alpha_{\max }+\theta_{0}<$ $180^{\circ}, " F$ " $<0$, and the interfacial energy, $E$, decreases monotonically as $h$ decreases (as the liquid penetrates the cavity-see Panel E). Therefore, the thermodynamic state of the cavities under the droplet is fully filled for any $\theta_{0}$. On the other hand, as illustrated in Panels $\mathrm{C}$ and $\mathrm{D}$, when $\alpha_{\max }+\theta_{0}>$ $180^{\circ}$, there must be a height, $h$, where the liquid-vapor interface flattens, " $F$ " $=0$ (i.e., $d E / d h=0$ ). At that height a local or global minimum energy is reached, as shown in Panel E. Based on the geometry of the cavity, $A_{\mathrm{LS}}$ and $A_{\mathrm{LV}}$ (shown in Panels $\mathrm{B}, \mathrm{C}$ and D) can be calculated and inserted into the "wetting equation", eq 10.

interfacial energy does not change with $h\left(\frac{d E}{d h}=0\right)$ and " $F$ " $=0$. In the absence of external forces, the liquid-vapor interface is stable (neither penetrating nor ejecting out of the cavity) in the case of Condition V.

In general, as illustrated in Figure 5B, the liquid-vapor interface in a cone-shaped cavity adopts a spherical curvature, and the interfacial force, " $F$ ", points toward the center of the sphere. " $F$ " is 0 (zero) only when $\alpha+\theta_{0}=180^{\circ}$, i.e., when the liquid-vapor interface is flat. The direction of " $F$ " determines whether the liquid would penetrate/condense $(" F "<0)$ or eject/evaporate ("F" $F 0$ ) into or out of any given cavity/pore.

2.3. Demonstration of How the "Local Force Equation" Can Be Used to Calculate $\phi_{\mathrm{LS}}, \phi_{\mathrm{LV}}, \phi_{\mathrm{SV}}^{0}$, and $\boldsymbol{\phi}_{\mathrm{Lv}}^{0}$ and, thus, $\boldsymbol{\theta}_{\mathrm{t}}$. In this section we demonstrate how the "local force equation" can be used to calculate the different area fractions, $\phi_{\mathrm{LS}}\left(=\phi_{1 \mathrm{~S}}\right), \phi_{\mathrm{LV}}\left(=\phi_{12}\right), \phi_{\mathrm{SV}}^{\mathrm{O}}\left(=\phi_{12}^{\mathrm{O}}\right)$, and $\phi_{\mathrm{LV}}^{\mathrm{O}}(=$ $\left.\phi_{12}^{\mathrm{O}}\right)$, on any type of texture. These area fractions can then be inserted into the "wetting equation" (eq 10) to calculate the contact angle on the textured surface, $\theta_{\mathrm{t}}$.

For this demonstration, let us consider a droplet on a textured surface, where the texture consists of spherical cavities, as illustrated in Figure 6. In contrast to the cone-shaped cavity, discussed above, the slope, $\alpha$, of the spherical cavity changes with respect to the cavity height, $h$ (see Figures $6 \mathrm{~B}, \mathrm{C}$, and D). Based on the "local force equation" one can conclude that the liquid-vapor interface would be metastable or thermodynamically stable at height $(\mathrm{s}), h$, where " $F "=0$ (i.e., $d E / d h=0)$. At this height(s), $\alpha+\theta_{0}=180^{\circ}$, and the liquid-vapor interface is flat.

In practice, in order to determine whether a cavity can be filled spontaneously (i.e., without an energy barrier), it is easiest to define the maximum slope of any tangent drawn in the cavity, $\alpha_{\max }$ as a reference (see example for $\alpha_{\max }$ in Figures 6B, $\mathrm{C}$, and D). We note (see Figures $6 \mathrm{~B}$ and D) that by definition the slope, $\alpha$, is zero at $h=0$ (bottom of the cavity) as well as at $h=H$ (top of the cavity).

When $\alpha_{\max }+\theta_{0}<180^{\circ}$, as illustrated in Figure 6B, at any $h$ the liquid-vapor interface is concave. Therefore, based on the "local force equation", the interfacial energy, E, decreases monotonically as the liquid penetrates the cavity (see the dashed line in Figure $6 \mathrm{E}$ ). As a result, the cavities under the droplet get filled spontaneously, $\phi_{\mathrm{LV}}=0$ (no liquid-vapor interface below the droplet) and $\phi_{\mathrm{LS}}=\phi_{\mathrm{LS}(\max )}$. Under these conditions the contact angle is given by eq 13 or eq 17 for "fully filled I empty" or "fully filled I partially filled" wetting states, respectively. To determine which wetting state takes place-the "fully filled I empty" or the "fully filled I partially filled"-the state of the cavities outside the droplet (empty or partially filled) should be determined, as elaborated below.

On the other hand, for $\alpha_{\max }+\theta_{0} \geq 180^{\circ}$ as illustrated in Figure $6 \mathrm{C}$ for $\theta_{0}<90^{\circ}$ and Figure $6 \mathrm{D}$ for $\theta_{0} \geq 90^{\circ}$, there would 


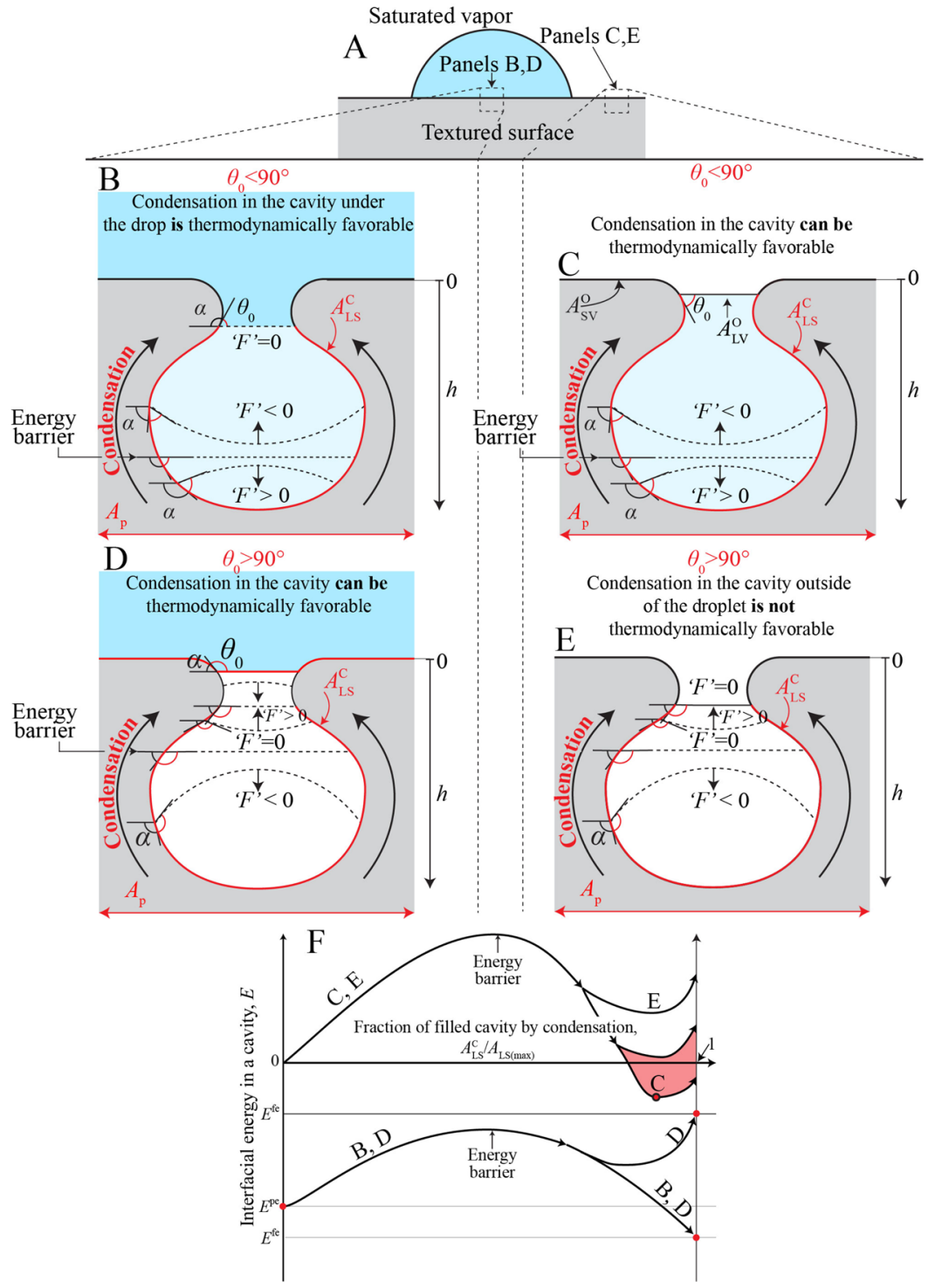

Figure 7. Schematic illustration that shows one path, out of many possible paths, by which the cavities under (Panels A, B, and D) and outside the droplet (Panels A, C, and E) can get filled by condensation. For the sake of simplicity, the illustration shows that condensation starts from the bottom of the cavity; however, in isotropic cavities, e.g., spherical cavity, condensation can initiate from any side of the cavity. Panel F shows a qualitative plot of the interfacial energy, $E$, vs the fraction of the filled cavity.

always be at least one height, $h$, where the liquid-vapor interface in the cavity flattens and $E$ reaches its local or global minimum. At that height, $\phi_{\mathrm{LS}}$ and $\phi_{\mathrm{LV}}$ can be geometrically calculated and inserted into eq 10 or eq 12 to calculate $\theta_{\mathrm{t}}^{\mathrm{pp}}$ (for partially filled cavities outside the droplet) or $\theta_{t}^{\text {pe }}$ (for empty cavities outside the droplet), respectively. As elaborated below, the "partially filled I partially filled" wetting state can only be metastable; however, as the dashed lines in Figure $6 \mathrm{E}$ show, the "partially filled I empty" wetting state can be either metastable or thermodynamically stable. To distinguish between a metastable and a thermodynamically stable "partially filled I empty" wetting state, a "total interfacial energy analysis" is discussed in the next section.

Apart from liquid penetration from the droplet side, since the vapor under the droplet is saturated, the cavities can get filled by condensation as illustrated in Figure 7 . It is important to note that we defined the liquid-solid contact area (under and outside the droplet) that forms due to condensation as $A_{\mathrm{LS}}^{\mathrm{C}}$ (the superscript "C" stands for "condensed"), which should not be confused with $A_{\mathrm{LS}}$ (liquid-solid area due to liquid that penetrates from the droplet). $A_{\mathrm{LS}}^{\mathrm{C}}$ should not be included for calculating $\phi_{\mathrm{LS}}\left(=\frac{A_{\mathrm{LS}}}{A_{\mathrm{P}}}\right)$, unless the condensed liquid comes into contact with the droplet. This is because until the condensed liquid coalesces with the main droplet it does not contribute to the droplet free energy or the interfacial tensions at the solidliquid-vapor interface.

Figures $7 \mathrm{~B}$ and $\mathrm{D}$ show one path (out of many possible paths) of how the cavities under the droplet can be filled by condensation. First, at a given thermal energy, a small droplet can condense in the cavity. Since the liquid-vapor interface of that condensed droplet is convex, i.e., " $F$ " $<0$, the interfacial tension resists further condensation; however, there is an energy barrier where the liquid-vapor interface flattens (see "energy barrier" in Figure 7B, where " $F$ " $=0$ ). Once the energy barrier is crossed, additional condensation renders the "local force", " $F$ ", positive, hence further condensation is energetically favorable. For the case of $\theta_{0}<90^{\circ}$, additional condensation 


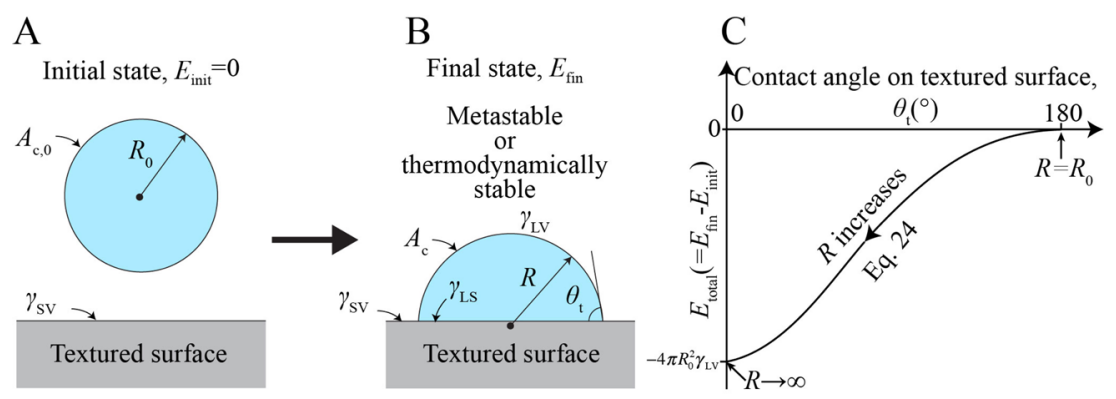

Figure 8. Total interfacial energy of a droplet on a textured surface, $E_{\text {total }}$, was derived by considering the two extreme states depicted in Panels A and B. Initially, the droplet is far apart from the textured surface, depicted in Panel A. Then the final state is depicted in Panel B, where the droplet is on the surface and has reached its metastable or thermodynamically stable state. Panel C shows a plot of eq 24 , indicating that $E_{\text {total }}$ decreases monotonically as the contact angle on the textured surface, $\theta_{\mathrm{t}}$, decreases and as the radius of the droplet on the surface, $R$, increases (i.e., $E_{\text {total }}$ decreases as the droplet spreads on the surface).

beyond the energy barrier reduces the surface energy until the cavity is completely filled. Interestingly, for any geometry, as discussed in the next section, when $\theta_{0}<90^{\circ}$, a filled cavity under the droplet is always the thermodynamically stable case (see Figure 7F; the interfacial energy of the cavity depicted in Panel B reaches the global minimum when the cavity is full). On the other hand, for $\theta_{0}>90^{\circ}$ (Figure $7 \mathrm{D}$ and energy profile is plotted in Figure $7 \mathrm{~F}$ ), the filled cavity under the droplet can be either metastable or thermodynamically stable. Again, the conditions in which the filled cavity is thermodynamically favorably stable are discussed in the next section.

In addition to condensation under the droplet, the cavities outside the droplet can also be filled by condensation if the vapor is saturated, which is often the case in close proximity to the droplet. As illustrated in Figures 7C and E, the "local force equation" shows that the liquid-vapor interface outside the droplet, $A_{\mathrm{LV}}^{\mathrm{O}}$ (the superscript "O" stands for "outside the droplet"), would be metastable or thermodynamically stable where $\alpha+\theta_{0}=180^{\circ}$. One can show (see Supporting Information Section 4) that for $\theta_{0}>90^{\circ}$ (Figure 7E) the cavities outside the droplet are empty (filled with vapor) at thermodynamic equilibrium for any type/shape of cavity, but if $\theta_{0}<90^{\circ}$ (Figure 7C) then partially filled (with liquid) cavities outside the droplet are thermodynamically stable if $\cos \theta_{0}>$ $A_{\mathrm{LV}}^{\mathrm{O}} / A_{\mathrm{LS}}^{\mathrm{O}}$, where $A_{\mathrm{LV}}^{\mathrm{O}}$ and $A_{\mathrm{LS}}^{\mathrm{O}}$ are the areas of the liquid-vapor and liquid-solid interfaces outside the droplet, respectively (see Figure 7C).

To summarize this section, it was shown that for a cavity of any geometry one can design or measure (e.g., by SEM) the maximum slope of the cavity, $\alpha_{\max }$. If $\theta_{0}+\alpha_{\max }<180^{\circ}$, there is no energy barrier for the liquid to spontaneously/completely fill the cavities under the droplet. In this case, $\theta_{\mathrm{LS}(\max )}$ can be calculated based on the geometry of the cavity and eq 14 (for empty cavities outside the droplet, $\left.\cos \theta_{0}<A_{\mathrm{LV}}^{\mathrm{O}} / A_{\mathrm{LS}}^{\mathrm{O}}\right)$. However, if the cavities outside get filled by condensation, i.e., $\cos \theta_{0}>$ $A_{\mathrm{LV}}^{\mathrm{O}} / A_{\mathrm{LS}}^{\mathrm{O}}$, the "fully filled I partially filled" wetting state is the thermodynamically stable case, and eq 17 should be used to calculate the macroscopic contact angle.

When $\theta_{0}+\alpha_{\max }>180^{\circ}$ the "partially filled I empty" wetting state can be metastable (could be stable for a long time-the determination of the stability time is beyond the scope of this paper), or in some cases as discussed below, it can be the thermodynamically stable wetting state. For calculating the contact angle for the "partially filled I empty" wetting state, $\theta_{\mathrm{t}}^{\mathrm{pe}}$, one should locate/design the height in the cavity, $h$, where $\alpha+$ $\theta_{0}=180^{\circ}$. At that height, where the liquid-vapor interface is flat, $\phi_{\mathrm{LS}}$ and $\phi_{\mathrm{LV}}$ can be calculated and inserted into eq 12 to get $\theta_{\mathrm{t}}^{\text {pe }}$. The "partially filled I partially filled" wetting state can only be a metastable state. Cavities outside the droplet can only get partially filled when $\theta_{0}<90^{\circ}$, and under this condition (see ref 53), the thermodynamic state of the cavities under the droplet is fully filled.

Lastly, it is not surprising that re-entrants, cavities that broaden below the surface, can stabilize the partially filled wetting state and render the surfaces omniphobic. By definition, re-entrant cavities exhibit $\alpha_{\max }>90^{\circ}$ (see Figure 1C, for instance). As $\alpha_{\max }$ increases (i.e., $\alpha_{\max }+\theta_{0}$ increases), the penetration into the cavities/pores of a liquid with low interfacial tension, such as oils, can be "stopped" by the capillary force, " $F$ ". Notably, when $\alpha_{\max }=180^{\circ}$, any liquid with $\theta_{0}>0$ (i.e., $\alpha_{\max }+\theta_{0}$ exceeds $180^{\circ}$ for any $\theta_{0}$ ) cannot penetrate the cavity with no energy barrier assuming that the effect of external forces is negligible in comparison to " $F$ ".

2.4. "Total Interfacial Energy Analysis" of a Droplet on a Textured Surface for Determining the Thermodynamically Stable Contact Angle. As discussed above, when $\alpha_{\max }+$ $\theta_{0}>180^{\circ}$ the "partially filled I empty" wetting state can be either metastable or thermodynamically stable. In this section, we consider the total interfacial energy of the droplet on a textured surface in order to distinguish between a metastable and thermodynamically stable "partially filled I empty" state.

To evaluate the total interfacial energy of a droplet on any textured surface, let us consider the initial and final states, as depicted in Figures $8 \mathrm{~A}$ and $\mathrm{B}$. In the initial state, Figure 8A, a droplet of radius $R_{0}$ is located far apart from a textured surface. Let us define the initial interfacial energy, $E_{\text {init }}$ as the reference state, where

$$
E_{\text {init }}=\gamma_{\mathrm{LV}} A_{\mathrm{C}, 0}+\gamma_{\mathrm{SV}} A_{\mathrm{LS}(\max )}
$$

where $A_{\mathrm{C}, 0}$ is the droplet liquid-vapor interfacial area (see Figure $8 \mathrm{~A}$ ) and $A_{\mathrm{LS}(\max )}$ is the real area of the textured surface.

In the final state, when the droplet contacts the textured surface, Figure $8 \mathrm{~B}$, a contact angle $\theta_{\mathrm{t}}$ (of any wetting state) is formed, which can be either metastable or thermodynamically stable. The energy at the final state is given by

$$
E_{\text {fin }}=\gamma_{\mathrm{LV}}\left(A_{\mathrm{C}}+A_{\mathrm{LV}}\right)+\gamma_{\mathrm{LS}} A_{\mathrm{LS}}+\gamma_{\mathrm{SV}}\left(A_{\mathrm{LS}(\max )}-A_{\mathrm{LS}}\right)
$$

where $A_{\mathrm{C}}$ is the curved area of the droplet on the surface as shown in Figure 8B.

Assuming that the droplet is a truncated sphere, the total interfacial change in energy can be calculated as follows (see 


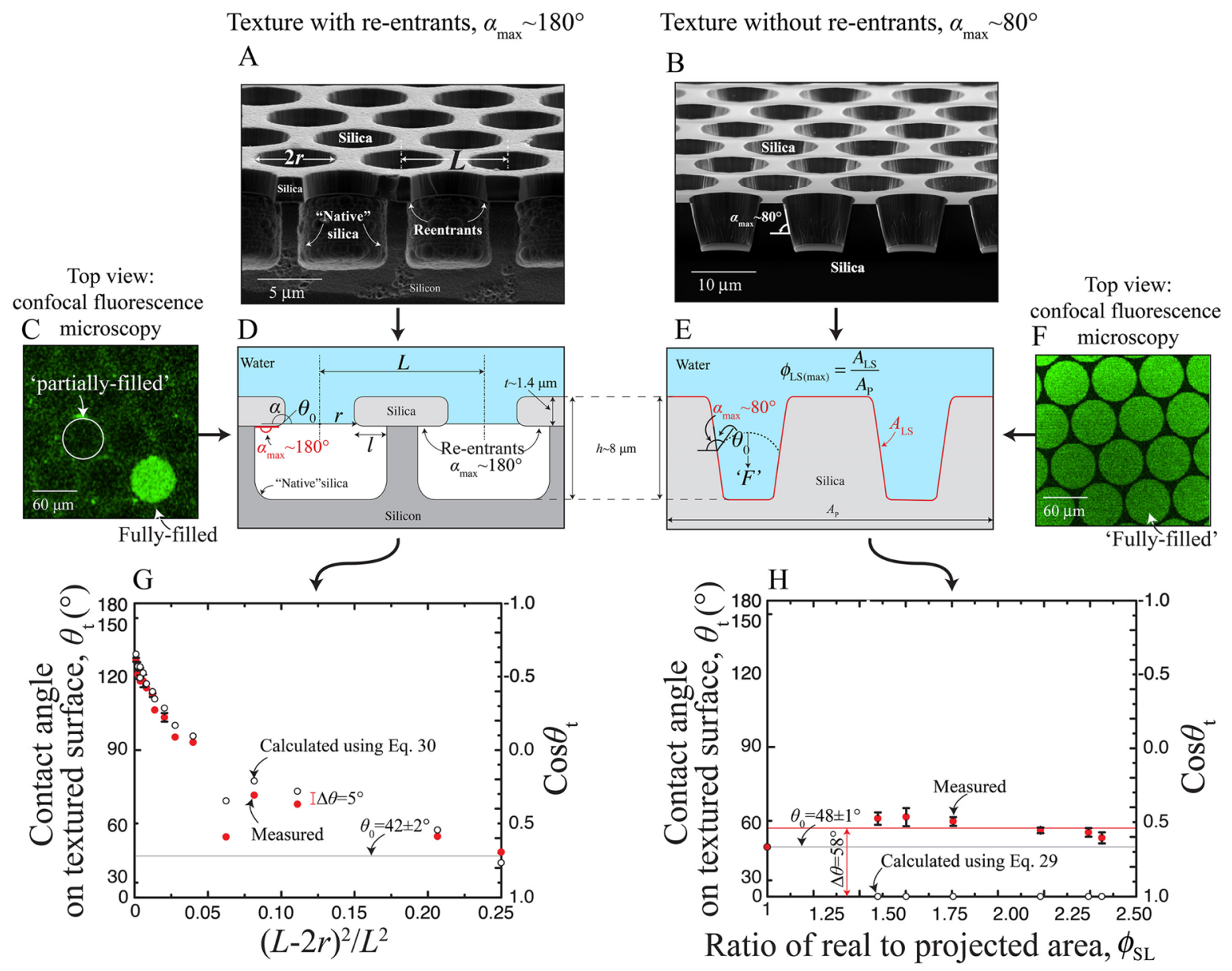

Figure 9. Experimental demonstration of the "wetting model". Two types of silica $\left(\theta_{0} \sim 45^{\circ}\right)$ textures were prepared: (1) re-entrant cavities, where $\alpha_{\max } \sim 180^{\circ}$, as shown in Panels A and D, and (2) non-re-entrant cavities, where $\alpha_{\max } \sim 80^{\circ}$, as shown in Panels B and E. The "wetting model" predicts that when $\alpha_{\max }+\theta_{0}>180^{\circ}$ and $\theta_{0}<90^{\circ}$, such as the case of deionized water on the re-entrant cavities, the "partially filled I empty" (CassieBaxter) wetting state is metastable. Using fluorescently dyed deionized water, the confocal microscopy image in Panel C indeed confirms that the majority of the re-entrant cavities under the liquid remained partially filled. On the other hand, when $\alpha_{\max }+\theta_{0}<180^{\circ}$, such as in the case of deionized water on the non-re-entrant cavities, liquid spontaneously (no energy barrier) fills the cavities, and the "fully filled I empty" (Wenzel state) wetting state is formed. Panel F indeed confirms that all the cavities under the droplet were filled within less than a minute after the droplet was deposited on the samples. Panels $\mathrm{G}$ and $\mathrm{H}$ show the measured contact angles, $\theta_{\mathrm{t}}$ and the calculated (not fitted) contact angles for cavities with (Panel G, eq 29) and without (Panel H, eq 28) re-entrants.

detailed derivation in the Supporting Information Section 5, which is based on Tadmor's work ${ }^{16}$ )

$$
\begin{aligned}
E_{\text {total }} & =E_{\text {fin }}-E_{\text {init }} \\
& =\frac{4 \pi R_{0}^{2} \gamma_{\mathrm{LV}}}{4^{1 / 3}\left(2-3 \cos \theta_{\mathrm{t}}+\cos ^{3} \theta_{\mathrm{t}}\right)^{-1 / 3}}-4 \pi R_{0}^{2} \gamma_{\mathrm{LV}}
\end{aligned}
$$

Figure $8 \mathrm{C}$ shows a plot of eq 24, indicating that the total interfacial energy of a droplet on any textured surface decreases monotonically as $\theta_{\mathrm{t}}$ decreases and $R$ increases (i.e., as the droplet spreads on the surface). Therefore, among the different contact angles, in which $d E / d h=-" F "=0$, the smallest contact angle, $\theta_{\mathrm{t}}$ is the thermodynamically favorable one, which is in agreement with Patankar's derivation. ${ }^{54}$

Based on eq 24, one can conclude that to achieve a thermodynamically stable "fully filled I empty" wetting state $\theta_{t}^{\text {fe }}$ should be smaller than $\theta_{\mathrm{t}}^{\mathrm{pe}}$, where $\theta_{\mathrm{t}}^{\mathrm{fe}}$ and $\theta_{\mathrm{t}}^{\mathrm{pe}}$ are the contact angles on the textured surface of the "fully filled I empty" and the "partially filled I empty" wetting states, respectively.
Therefore, for the thermodynamically stable "fully filled I empty" wetting state

$$
\phi_{\mathrm{LS}(\max )} \cos \theta_{0}>\phi_{\mathrm{LS}} \cos \theta_{0}-\phi_{\mathrm{LV}}
$$

where $\cos \theta_{\mathrm{t}}^{\mathrm{fe}}=\phi_{\mathrm{LS}(\max )} \cos \theta_{0}$ (left side of eq 25) and $\cos \theta_{\mathrm{t}}^{\mathrm{pe}}=$ $\phi_{\mathrm{LS}} \cos \theta_{0}-\phi_{\mathrm{LV}}$ (right side of eq 25).

Considering the "local force equation" (eq 21), eq 25 can be summarized as follows: (1) for $\theta_{0}<90^{\circ}$ and $\cos \theta_{0}<A_{\mathrm{LV}}^{\mathrm{O}} / A_{\mathrm{LS}}^{\mathrm{O}}$ the "fully filled I empty" wetting state (Wenzel) is the thermodynamically stable state for any cavity type/shape. (2) If $\theta_{0}<90^{\circ}$ and $\cos \theta_{0}>A_{\mathrm{LV}}^{\mathrm{O}} / A_{\mathrm{LS}}^{\mathrm{O}}$, then the "fully filled I partially filled" wetting state is the thermodynamically favored state. (3) If $\theta_{0}>90^{\circ}$ (and since $\phi_{\mathrm{LS}(\max )}=\phi_{\mathrm{LS}}+\phi_{\mathrm{LV}}$ ), then the "fully filled I empty" wetting state is thermodynamically stable when

$$
\cos \theta_{0}>-\frac{A_{\mathrm{LV}}}{A_{\mathrm{SV}}}
$$

Lastly, (4) the "partially filled I empty" wetting state (CassieBaxter) is thermodynamically stable only when $\theta_{0}>90^{\circ}$ and $\alpha_{\max }+\theta_{0}>180^{\circ}$ and 


$$
\cos \theta_{0}<-\frac{A_{\mathrm{LV}}}{A_{\mathrm{SV}}}
$$

\section{EXPERIMENTAL DEMONSTRATION OF THE "WETTING MODEL"}

The effects of texture on contact angles have been experimentally studied before. ${ }^{3,6,11,14,17,19,46,47,55-62}$ In this section we explicitly focus on the effects of re-entrants and demonstrate how the equations of the "wetting model" can be easily applied.

For this demonstration, a series of contact angles of deionized water $(18.2 \mathrm{M} \Omega \mathrm{cm}$, Milli-Q) under atmospheric conditions, $\theta_{t}$, were measured on two different nano/microfabricated silica textures: (1) cavities with re-entrants (Figures $9 \mathrm{~A}$ and D), where $\alpha_{\max } \sim 180^{\circ}$, and (2) cavities without reentrants (Figures $9 \mathrm{~B}$ and $\mathrm{E}$ ), where $\alpha_{\max } \sim 80^{\circ}$. For each type of sample (cavities with and without re-entrants), a series of different array of cavities were prepared. Each array consisted of a specific opening radius, $r$ (from 1 to $30 \mu \mathrm{m}$ ), and a specific distance between two neighboring cavities, $L$ (from 2 to 62 $\mu \mathrm{m})$. Sample preparation and characterization are described in Section 6 of the Supporting Information.

Figures $9 \mathrm{G}$ and $\mathrm{H}$ show the measured and the calculated contact angles using eqs 28 and 29, which are elaborated below, for the samples with and without re-entrant cavities, respectively. Detailed descriptions of the contact angle measurements can be found in the Supporting Information Section 6.3. Additional measurements of contact angles of canola oil $\left(\gamma_{\mathrm{LV}} \sim 35 \mathrm{mN} / \mathrm{m}\right)^{63}$ on the re-entrant and non-reentrant cavities can be found in Section 6.5 of the Supporting Information, which show good agreement with the "wetting model”.

For the cavities without re-entrants, $\alpha_{\max }+\theta_{0}$ is smaller than $180^{\circ}$ (see left cavity in Figure 9E, $\theta_{0}=42 \pm 2^{\circ}$ ). Hence, based on eq 21 (see Summary and Conclusions section, Case I), the liquid-vapor interface is forced all the way to the bottom of the cavity (see also Condition 2 in Figure $S 8, \theta_{\mathrm{t}}<90^{\circ}$ ), and the "fully filled I empty" wetting state should form with no energy barrier to overcome. Confocal imaging, shown in Figure 9F (green circles show that the cavities were filled with deionized water with a fluorescent dye), confirms that the cavities under the droplet were fully filled within less than 5 minutes after droplet deposition. For this "fully filled I empty" wetting state, one can easily calculate the normalized liquid-solid area, $\phi_{\mathrm{LS}}$, and use eq 14 to predict $\theta_{t}^{\text {fe }}$ for the given geometry

$$
\cos \theta_{\mathrm{t}}^{\mathrm{fe}}=\phi_{\mathrm{SL}} \cos \theta_{0}=\left(1+\frac{4 \pi r h}{\sqrt{3} L^{2}}\right) \cos \theta_{0}
$$

where $r$ is the opening radius of the cavity; $h$ is the height of the cavity; and $L$ is the distance between two neighboring cavities (Figure 9E).

For the cavities with re-entrants $\alpha_{\max }+\theta_{0}$ exceeds $180^{\circ}$. The "wetting model" predicts (see Summary and Conclusions section, Case II) that the "partially filled I empty" wetting state should be metastable (see also Figure S8, Condition 1 for any $\left.\theta_{0}\right)$. The confocal image in Figure $9 \mathrm{C}$ confirms that the majority of the cavities below the droplet were partially filled. By examining the slopes of the re-entrant cavity, one can determine that $\alpha+\theta_{0}$ equals $180^{\circ}$ at the bottom of the overhanging layer (see Figure 9D); hence, the liquid-vapor interface is stable at the bottom of the overhanging layer. Once the penetration depth of the liquid-vapor interface is known, one can calculate $\phi_{\mathrm{LS}}^{\mathrm{pe}}$ and $\phi_{\mathrm{LV}}^{\mathrm{pe}}$ and use eq 12 (equivalent to the Cassie-Baxter model) to calculate the corresponding contact angle

$$
\cos \theta_{\mathrm{t}}^{\mathrm{pe}}=\left(1-\frac{\pi}{2 \sqrt{3}} \frac{4 r^{2}}{L^{2}}+\frac{4 \pi}{\sqrt{3}} \frac{r t}{L^{2}}\right) \cos \theta_{0}-\frac{\pi}{2 \sqrt{3}} \frac{4 r^{2}}{L^{2}}
$$

where $t$ is the thickness of the overhanging layer (Figure 9D).

The plot in Figure 9H shows that in the case of the non-reentrant cavities the average discrepancy, $\Delta \theta$, between the measured contact angle, $\theta_{\mathrm{t}}$, and the calculated contact angle, $\theta_{\mathrm{t}}^{\mathrm{fe}}$, was $58^{\circ}$. We note that all the calculated contact angles are smaller than the measured contact angles. This large discrepancy is due to dynamic effects-namely, advancing and receding contact angles (or contact angle hysteresis). In systems where the liquid has to advance to reach a metastable or thermodynamic equilibrium, the advancing contact angle must be larger than the intrinsic contact angle, $\theta_{0}$; otherwise, there is no driving force (the driving force is proportional to $\left.\cos \theta_{t}-\cos \theta_{0}\right)$ for the liquid to advance. ${ }^{64,65}$ On the other hand, for systems where the liquid has to recede in order to equilibrate, the macroscopic contact angle must be lower than the intrinsic contact angle. ${ }^{64,65}$

In the case of re-entrant cavities (Figure 9G), however, the average discrepancy, $\Delta \theta$, between the measured, $\theta_{t}$ and the calculated contact angle, $\theta_{\mathrm{t}}^{\text {pe }}$ (eq 22), was $5^{\circ}$. As expected, the contact angle increases as the liquid-vapor interface below the droplet increases (note that large $\phi_{\mathrm{LV}}$ is equivalent to ( $L-$ $2 r)^{2} / L^{2}$; see $x$ axes in Figure $9 \mathrm{G}$ ). In this case, since the measured contact angle, $\theta_{t}$, is significantly larger than the intrinsic contact angle, $\theta_{0}$, there is a driving force for the liquid to spread and reach its metastable state, as predicted by the "wetting model". Note, however, that since $\theta_{0}<90^{\circ}$ the contact angles that were measured for the re-entrant cavities are metastable. It is only a matter of time until all of the cavities under the droplet would eventually get filled. It is also straightforward to show (see Section 7 in the Supporting Information) that for all of the samples (with and without reentrants) the cavities outside the droplet would ultimately become partially filled in saturated vapor environment. Therefore, in saturated vapor, the "fully filled I partially filled" wetting state is the thermodynamically favorable wetting state for all of the samples in our experiments. Experimental studies of the dynamics of cavity filling under and outside droplets is beyond the scope of this paper.

It is important to note that the dissolution/evaporation rate of the vapor, as the liquid penetrated the cavities, was not the dominant mechanism for the cavity filling; the interfacial force, as predicted by eq 21 , dominated the cavity filling. Confocal images (Figures 9C and F) show that the re-entrant cavities remained "partially filled" for at least several hours, whereas the cavities without re-entrants got fully filled within less than a minute, even though in both cases-re-entrant and non-reentrant cavities - vapor had to evacuate from the cavities via a dissolution/evaporation mechanism, as the cavities are unconnected with one another. Experimentally, we also find that cavity filling can be achieved by the slow penetration of a thin wetting film along the walls of cavities, which pushes out the vapor phase that eventually detaches as a bubble (without dissolution/evaporation occurring at any stage of this process). 
We also note that the "wetting model" still holds even if the surfaces are chemically heterogeneous (e.g., "contaminated"), but so long as the areas of the heterogeneities are much smaller than the droplet area, which is often the case. Under these conditions, the intrinsic contact angle, $\theta_{0}$ - when averaged over the flat but chemically heterogeneous/contaminated surfaceleads to the Cassie or Israelachvili-Gee equations depending on whether the domains are microscopic or nanoscopic (molecular). ${ }^{38,66}$

\section{SUMMARY AND CONCLUSIONS}

In this paper we developed a simple-to-apply wetting model and compared its predictions with experimental results on surfaces with complex but realistic topologies. Effects of gravitational forces, Laplace pressure of the droplet, linetension, contact angle hysteresis, droplet impact velocity, and any other external forces are not considered in this model. Yet, our experiments quantitatively corroborated the predictions of the model given the experimental parameters.

Two general conclusions can be derived from our theoretical analysis and from our experimental demonstration:

(1) When the intrinsic contact angle, $\theta_{0}$, is smaller than $90^{\circ}$, any type/shape of nano/microcavities under the droplet will eventually get fully filled; thus, the thermodynamically stable wetting state can be either the "fully filled I empty" or the "fully filled I partially filled" wetting state. The corresponding thermodynamic contact angles, $\theta_{0}^{\mathrm{fe}}$ or $\theta_{0}^{\mathrm{fp}}$, are lower than $\theta_{0}$ for any texture, including for re-entrant cavities. Therefore, thermodynamically stable omniphobic surfaces are unrealistic when $\theta_{0}<90^{\circ}$. However, the "partially filled I empty" wetting state can be made metastable (the conditions are specified below), and the metastable $\theta_{\mathrm{t}}^{\text {pe }}$ can be as high as $180^{\circ}$ for any liquid (the so-called "omniphobic state"). The time, i.e., dynamics, at which the "partially filled I empty" wetting state eventually transforms to the "fully filled I empty" (i.e., the transition from Cassie-Baxter to Wenzel wetting states) or to the "fully filled I partially filled" wetting state will be reported in a subsequent paper; however, it is expected that the stability of the "partially filled I empty" wetting state would increase (be stable for a longer time) with the energy barrier that is required for liquid penetration and/or condensation. The energy barrier for liquid penetration (see Figures 6C and D) and condensation can be calculated for a given cavity by integrating the "local force equation" (eq 21) with respect to the liquid height, $h$, up to the height where the liquid-vapor interface is flat. As a design consideration, unconnected cavities are expected to stabilize the "partially filled I empty" wetting state better than connected cavities. The vapor (or Phase 2) in the case of connected cavities can flow under the liquid (or Phase 1) and evacuate faster than in the case of unconnected cavities.

(2) On the other hand, if $\theta_{0}$ is larger than $90^{\circ}$, then $\theta_{t}$ is larger than $90^{\circ}$ for any nano/microtexture. Therefore, texturedriven omniphilicity $\left(\theta_{t}<90^{\circ}\right.$ for any liquid) is not physically possible. For instance, the metastable or thermodynamically stable contact angle of deionized water on Teflon (smooth or textured) cannot be lower than $90^{\circ}$.

In order to calculate/predict the contact angle on an engineered textured surface, $\theta_{t}$ (Figure S8, Supporting Information), the "wetting model" requires three key input parameters: (i) the slope of the cavities, $\alpha$, as a function of the cavity depth, $h$ (which can be easily obtained by imaging); (ii) the intrinsic contact angle on smooth surface, $\theta_{0}$, and (iii) the surface area of the cavity (or the real area of the repeating unit cell), $\phi_{\mathrm{LS}(\max )}$. Once these three parameters are known, one can follow the procedure below in order to determine the wetting state, the contact angle on the textured surface, and the contact angle stability (metastable or thermodynamically stable):

(Case I) If $\alpha_{\max }+\theta_{0}<180^{\circ}$ (left and right panels in Figure S8, Condition 1) then the cavities under the droplet get filled with no energy barrier for any $\theta_{0}$. If the cavities outside the droplet are empty, i.e., $\cos \theta_{0}<A_{\mathrm{LV}}^{\mathrm{O}} / A_{\mathrm{LS}}^{\mathrm{O}}$, the contact angle of the "fully filled I empty" wetting state (Wenzel), $\theta_{\mathrm{t}}^{\mathrm{fe}}$, is the thermodynamically stable state, which can be calculated using eq 14. If the cavities outside the droplet are partially filled, i.e., $\cos \theta_{0}>A_{\mathrm{LV}}^{\mathrm{O}} / A_{\mathrm{LS}}^{\mathrm{O}}$, then the "fully filled I partially filled" wetting state is the thermodynamically stable state, and eq 17 should be used to calculate the true equilibrium contact angle, $\theta_{\mathrm{t}}^{\mathrm{fp}}$.

(Case II) When $\theta_{0}<90^{\circ}$ and $\alpha_{\max }+\theta_{0}>180^{\circ}$ (right panel in Figure S8, Condition 2), there must be a height, $h$, or several heights, where $\alpha+\theta_{0}=180^{\circ}$. At this height(s) the partially empty (under the droplet, i.e., Cassie-Baxter) wetting state is metastable, which can be stable for seconds to years. For each of these heights, where $\alpha+\theta_{0}=180^{\circ}$, the liquid-vapor interface is flat. $\phi_{\mathrm{LS}}$ and $\phi_{\mathrm{LV}}$ can then be calculated and used in eq 12 to calculate the metastable contact angle, $\theta_{\mathrm{t}}^{\text {pe }}$. If the cavities outside the droplet are empty (i.e., $\cos \theta_{0}<A_{\mathrm{LV}}^{\mathrm{O}} / A_{\mathrm{LS}}^{\mathrm{O}}$ ), then the thermodynamic contact angle (lower than $\theta_{0}$ ) is the "fully filled I empty" one, $\theta_{\mathrm{t}}^{\mathrm{fe}}$, which can be calculated using eq 14 (Wenzel). If the cavities outside the droplet are partially filled, i.e., $\cos \theta_{0}>A_{\mathrm{LV}}^{\mathrm{O}} / A_{\mathrm{LS}}^{\mathrm{O}}$, then the "fully filled I partially filled" contact angle, $\theta_{\mathrm{t}}^{\mathrm{fp}}$, is thermodynamically stable, which can be calculated using eq 17 .

(Case III) If $\theta_{0}>90^{\circ}$ and $\alpha_{\max }+\theta_{0}>180^{\circ}$ (left panel in Figure S8, Conditions 2 and 3 ) then the case is similar to that discussed in Case (II); however, the "partially filled I empty" wetting state is a thermodynamically stable state if $\cos \theta_{0}<-\frac{A_{\mathrm{LV}}}{A_{\mathrm{SV}}}$. We note that this is the only case where the "partially filled I empty" wetting state is the thermodynamically favorable state. We note also that in this case the contact angle of the "partially filled I empty" state is smaller than the contact angle of the "fully filled I empty" wetting state $\left(\theta_{\mathrm{t}}^{\mathrm{pe}}<\theta_{\mathrm{t}}^{\mathrm{fe}}\right)$. The "fully filled I empty" contact angle, $\theta_{\mathrm{t}}^{\mathrm{fe}}$, is thermodynamically stable if $\cos \theta_{0}>-\frac{A_{\mathrm{LV}}}{A_{\mathrm{SV}}}$.

\section{ASSOCIATED CONTENT}

\section{Supporting Information}

The Supporting Information is available free of charge on the ACS Publications website at DOI: 10.1021/acs.jpcc.7b00003.

Estimate of the droplet Laplace pressure and hydrostatic pressure and their effect of the apparent contact angle on textured surface; Supporting Information for the derivation of the "wetting equation"; Criterion for liquid condensation in the cavities outside the droplet; Derivation of the total interfacial energy of a droplet on textured surface; Description of the preparation of the textured surfaces; Supporting Information for the experimental section; and Figure that summarizes the "wetting model" (PDF)

\section{AUTHOR INFORMATION}

\section{Corresponding Author}

*E-mail: yairkau@bgu.ac.il. Phone: +972 (52) 6677228. 


\section{ORCID}

Yair Kaufman: 0000-0001-7771-8502

Szu-Ying Chen: 0000-0003-3486-9698

\section{Author Contributions}

$\triangle$ Y.K. and S.-Y.C. contributed equally to this work.

\section{Notes}

The authors declare no competing financial interest.

\section{ACKNOWLEDGMENTS}

This work was supported by a grant from the Procter \& Gamble Company. Himanshu Mishra was supported by an Elings Prize Fellowship in Experimental Science of the California NanoSystems Institute at the University of California, Santa Barbara. Stephen H. Donaldson was also supported by LabEX ENS-ICFP: ANR-10-LABX-0010/ANR10-IDEX-0001-02 PSL*. Dong Woog Lee was supported by grants from the National Research Foundation of Korea funded by the Korean Government (NRF-2016R1C1B2014294).

\section{REFERENCES}

(1) Nosonovsky, M. Multiscale Roughness and Stability of Superhydrophobic Biomimetic Interfaces. Langmuir 2007, 23, 31573161.

(2) Nosonovsky, M.; Bhushan, B. Why Re-Entrant Surface Topography Is Needed for Robust Oleophobicity. Philos. Trans. $R$. Soc., A 2016, 374, 20160185-13.

(3) Feng, L.; Zhang, Y.; Xi, J.; Zhu, Y.; Wang, N.; Xia, F.; Jiang, L. Petal Effect: a Superhydrophobic State with High Adhesive Force. Langmuir 2008, 24, 4114-4119.

(4) Tsai, C.-C.; Gu, Y.; Kornev, K. G. Wetting of Nanofiber Yarns. Colloids Surf., A 2014, 459, 22-30.

(5) Quéré, D. Non-Sticking Drops. Rep. Prog. Phys. 2005, 68, 24952532 .

(6) Butt, H.-J.; Vollmer, D.; Papadopoulos, P. Super Liquid-Repellent Layers: the Smaller the Better. Adv. Colloid Interface Sci. 2015, 222, 104-109.

(7) Mishra, H.; Schrader, A. M.; Lee, D. W.; Gallo, A.; Chen, S.-Y.; Kaufman, Y.; Das, S.; Israelachvili, J. N. Time-Dependent Wetting Behavior of PDMS Surfaces with Bioinspired, Hierarchical Structures. ACS Appl. Mater. Interfaces 2016, 8, 8168-8174.

(8) Hejazi, V.; Nyong, A. E.; Rohatgi, P. K.; Nosonovsky, M. Wetting Transitions in Underwater Oleophobic Surface of Brass. Adv. Mater. 2012, 24, 5963-5966.

(9) Milne, A. J. B.; Amirfazli, A. The Cassie Equation: How It Is Meant to Be Used. Adv. Colloid Interface Sci. 2012, 170, 48-55.

(10) Nosonovsky, M. Materials Science: Slippery When Wetted. Nature 2011, 477, 412-413.

(11) Choi, W.; Tuteja, A.; Mabry, J. M.; Cohen, R. E.; McKinley, G. H. A Modified Cassie-Baxter Relationship to Explain Contact Angle Hysteresis and Anisotropy on Non-Wetting Textured Surfaces. J. Colloid Interface Sci. 2009, 339, 208-216.

(12) Extrand, C. W. Model for Contact Angles and Hysteresis on Rough and Ultraphobic Surfaces. Langmuir 2002, 18, 7991-7999.

(13) Bormashenko, E. Physics of Solid-Liquid Interfaces: From the Young Equation to the Superhydrophobicity (Review Article). Low Temp. Phys. 2016, 42, 622-635.

(14) Tuteja, A.; Choi, W.; Ma, M.; Mabry, J. M.; Mazzella, S. A.; Rutledge, G. C.; McKinley, G. H.; Cohen, R. E. Designing Superoleophobic Surfaces. Science 2007, 318, 1618-1622.

(15) Bhushan, B.; Jung, Y. C. Natural and Biomimetic Artificial Surfaces for Superhydrophobicity, Self-Cleaning, Low Adhesion, and Drag Reduction. Prog. Mater. Sci. 2011, 56, 1-108.

(16) Tadmor, R. Line Energy and the Relation Between Advancing, Receding, and Young Contact Angles. Langmuir 2004, 20, 7659-7664.
(17) Tuteja, A.; Choi, W.; Mabry, J. M.; McKinley, G. H.; Cohen, R. E. Robust Omniphobic Surfaces. Proc. Natl. Acad. Sci. U. S. A. 2008, 105, 18200-18205.

(18) Multanen, V.; Chaniel, G.; Grynyov, R.; Loew, R. Y.; Siany, N. K.; Bormashenko, E. Hydrophilization of Liquid Surfaces by Plasma Treatment. Colloids Surf., A 2014, 461, 225-230.

(19) Kota, A. K.; Choi, W.; Tuteja, A. Superomniphobic Surfaces: Design and Durability. MRS Bull. 2013, 38, 383-390.

(20) Hensel, R.; Helbig, R.; Aland, S.; Braun, H.-G.; Voigt, A.; Neinhuis, C.; Werner, C. Wetting Resistance at Its Topographical Limit: the Benefit of Mushroom and Serif $\mathrm{T}$ Structures. Langmuir 2013, 29, 1100-1112.

(21) Liu, T. L.; Kim, C.-J. C. J. Repellent Surfaces. Turning a Surface Superrepellent Even to Completely Wetting Liquids. Science 2014, 346, 1096-1100.

(22) Bhushan, B. Biomimetics: Bioinspired Hierarchical-Structured Surfaces for Green Science and Technology 2016, DOI: 10.1007/978-3319-28284-8.

(23) Jiang, H.-R.; Chan, D.-C. Superhydrophobicity on Nanostructured Porous Hydrophilic Material. Appl. Phys. Lett. 2016, 108, $171603-171605$

(24) Li, Z.; Liao, K.; Liao, F.; Xiao, Q.; Jiang, F.; et al. Wetting and Spreading Behaviors of Nanodroplets: the Interplay Among Substrate Hydrophobicity, Roughness, and Surfactants. J. Phys. Chem. C 2016, 120, 15209-15215.

(25) Lv, C.; Hao, P.; Zhang, X.; He, F. Drop Impact Upon Superhydrophobic Surfaces with Regular and Hierarchical Roughness. Appl. Phys. Lett. 2016, 108, 141602-141605.

(26) Ennaceri, H.; Wang, L.; Erfurt, D.; Riedel, W.; Mangalgiri, G.; Khaldoun, A.; Kenz; El, A.; Benyoussef, A.; Ennaoui, A. WaterResistant Surfaces Using Zinc Oxide Structured Nanorod Arrays with Switchable Wetting Property. Surf. Coat. Technol. 2016, 299 (C), 169176.

(27) Ramiasa-MacGregor, M.; Mierczynska, A.; Sedev, R.; Vasilev, K. Tuning and Predicting the Wetting of Nanoengineered Material Surface. Nanoscale 2016, 8 (8), 4635-4642.

(28) Jardim, P. L. G.; Horowitz, F.; Felde, N.; Schröder, S.; Coriand, L.; Duparré, A. Determination of the Wenzel Roughness Parameter by the Power Spectral Density of Functional Alumina Surfaces. Thin Solid Films 2016, 606 (C), 57-62.

(29) Young, T. An Essay on the Cohesion of Fluids. Philos. Trans. $R$. Soc. London 1805, 95, 65.

(30) Wenzel, R. N. Resistance of Solid Surfaces to Wetting by Water. Ind. Eng. Chem. 1936, 28, 988-994.

(31) Amabili, M.; Lisi, E.; Giacomello, A.; Casciola, C. M. Wetting and Cavitation Pathways on Nanodecorated Surfaces. Soft Matter 2016, 12, 3046-3055.

(32) Marmur, A. From Hygrophilic to Superhygrophobic: Theoretical Conditions for Making High-Contact-Angle Surfaces From LowContact-Angle Materials. Langmuir 2008, 24, 7573-7579.

(33) Marmur, A. Hydro- Hygro- Oleo- Omni-Phobic? Terminology of Wettability Classification. Soft Matter 2012, 8, 6867-5.

(34) Drelich, J.; Marmur, A. Physics and Applications of Superhydrophobic and Superhydrophilic Surfaces and Coatings. Surf. Innovations 2013, 2, 211-227.

(35) Bormashenko, E. General Equation Describing Wetting of Rough Surfaces. J. Colloid Interface Sci. 2011, 360, 317-319.

(36) Both fluids, Phase 1 and Phase 2, must not dissolve the solid substrate, "Phase $S$ ", as depicted in Figure 2. In the case of nonconnected/closed cavities, miniscule miscibility of "Phase 2" in "Phase 1" is required in order to allow the penetration of "Phase 1" into the cavities and evacuation of "Phase 2 " from the cavities via dissolution of "Phase 1".

(37) Each wetting state is described by two terms: the first term describes the wetting state of the cavities under the liquid droplet, and the second describes the cavities outside the liquid droplet. The name format of the wetting states is "the state of the cavities under the droplet I the state of the cavities outside the droplet". Consequently, there are four different wetting states: (1) The "fully filled I empty" 
wetting state (Figures $3 \mathrm{E}$ and $\mathrm{F}$ ), where the cavities under the droplet are fully filled with Phase 2 (or liquid, for the case of solid-liquidliquid system) and the cavities outside the droplet are empty (i.e., filled with Phase 2 or vapor). For the "fully filled I empty" wetting state, the contact angle is $\theta_{\mathrm{t}}^{\mathrm{fe}}$, and this wetting state is equivalent to the Wenzel state. (2) The "partially filled I empty" wetting state (Figures 3B and $\mathrm{C})$, which is equivalent to the Cassie-Baxter state and the contact angle is $\theta_{\mathrm{t}}^{\mathrm{pe}}$. (3) "Partially filled I partially filled" (Figure $3 \mathrm{~A}$ ), where the contact angle is $\theta_{\mathrm{t}}^{\mathrm{pp}}$, and (4) the "fully filled I partially filled" wetting state (Figure $3 \mathrm{~F}$ ), where the contact angle is $\theta_{\mathrm{t}}^{\mathrm{fp}}$. Note that a cavity is considered fully filled if there is no liquid-vapor (or liquid-liquid) interface anywhere within cavity.

(38) Cassie, A. B. D.; Baxter, S. Wettability of Porous Surfaces. Trans. Faraday Soc. 1944, 40, 546-546.

(39) Marmur, A.; Bittoun, E. When Wenzel and Cassie Are Right: Reconciling Local and Global Considerations. Langmuir 2009, 25, $1277-1281$.

(40) Marmur, A. From Hygrophilic to Superhygrophobic: Theoretical Conditions for Making High-Contact-Angle Surfaces From LowContact-Angle Materials. Langmuir 2008, 24, 7573-7579.

(41) Whyman, G.; Bormashenko, E.; Stein, T. The Rigorous Derivation of Young, Cassie-Baxter and Wenzel Equations and the Analysis of the Contact Angle Hysteresis Phenomenon. Chem. Phys. Lett. 2008, 450, 355-359.

(42) Tadmor, R. Line Energy, Line Tension and Drop Size. Surf. Sci. 2008, 602, 108-111.

(43) Marmur, A. Line Tension and the Intrinsic Contact Angle in Solid-Liquid-Fluid Systems. J. Colloid Interface Sci. 1997, 186, 462466.

(44) Rykaczewski, K.; Paxson, A. T.; Staymates, M.; Walker, M. L.; Sun, X.; Anand, S.; Srinivasan, S.; McKinley, G. H.; Chinn, J.; Scott, J. H. J.; et al. Dropwise Condensation of Low Surface Tension Fluids on Omniphobic Surfaces. Sci. Rep. 2014, 4, 1-8.

(45) Quéré, D. Wetting and Roughness. Annu. Rev. Mater. Res. 2008, $38,71-99$.

(46) Wang, Q.; Yao, X.; Liu, H.; Quéré, D.; Jiang, L. Self-Removal of Condensed Water on the Legs of Water Striders. Proc. Natl. Acad. Sci. U. S. A. 2015, 112, 9247-9252.

(47) Bico, J.; Thiele, U.; Quéré, D. Wetting of Textured Surfaces. Colloids Surf., A 2002, 206, 41-46.

(48) Israelachvili, J. N. Intermolecular and Surface Forces: Revised Third Edition 2011, 151.

(49) Tsori, Y. Discontinuous Liquid Rise in Capillaries with Varying Cross-Sections. Langmuir 2006, 22, 8860-8863.

(50) Tsori, Y. Discontinuous Meniscus Location in Tapered Capillaries Driven by Pressure Difference and Dielectrophoretic Forces. Langmuir 2007, 23, 8028-8034.

(51) It is easy to show that any correction term due to a high local change in geometry, e.g., change in radius of curvature, $r^{*}$, results in correction terms for the energy of order $d h / r^{*}$ (i.e., zero). In other words, for any finite local curvature or change in geometry of magnitude $r^{*}$, however small, as $d h \rightarrow 0, r^{*}$ has no effect on the total energy change. Therefore, the change in energy is the same as for $r=$ $\infty$, which is equivalent to a locally flat sloping surface (of constant $\alpha$ ) as in Figure 4.

(52) This being the (unresolved) force normal to the surface, the $\gamma_{\mathrm{LV}}$ $\sin \theta_{0}$ component of the liquid-vapor interfacial tension in the Young equation.

(53) This metastable state can arise when droplet advances or recedes, leaving partially filled cavities outside the droplet before the cavities under the droplet have become filled. These effects will be determined by such time-dependent experimental parameters as the time between advancing and receding, the relative rates of vapor condensation into the bulk liquid, and capillary condensation into the cavities.

(54) Patankar, N. A. On the Modeling of Hydrophobic Contact Angles on Rough Surfaces. Langmuir 2003, 19, 1249-1253.
(55) Amabili, M.; Giacomello, A.; Meloni, S. Unraveling the Salvinia Paradox: Design Principles for Submerged Superhydrophobicity. Adv. Mater. Interfaces 2015, 2, 1-5.

(56) Shibuichi, S.; Onda, T.; Satoh, N. Super Water-Repellent Surfaces Resulting From Fractal Structure. J. Phys. Chem. 1996, 100, 19512-19517.

(57) Johnson, R. E., Jr; Dettre, R. H. Contact Angle Hysteresis. III. Study of an Idealized Heterogeneous Surface. J. Phys. Chem. 1964, 68, $1744-1750$

(58) Peters, A. M.; Pirat, C.; Sbragaglia, M.; Borkent, B. M.; Wessling, M.; Lohse, D.; Lammertink, R. G. H. Cassie-Baxter to Wenzel State Wetting Transition: Scaling of the Front Velocity. Eur. Phys. J. E: Soft Matter Biol. Phys. 2009, 29, 391-397.

(59) Manukyan, G.; Oh, J. M.; van den Ende, D.; Lammertink, R. G. H.; Mugele, F. Electrical Switching of Wetting States on Superhydrophobic Surfaces: a Route Towards Reversible Cassie-to-Wenzel Transitions. Phys. Rev. Lett. 2011, 106, 014501-014504.

(60) Bonn, D.; Eggers, J.; Indekeu, J.; Meunier, J.; Rolley, E. Wetting and Spreading. Rev. Mod. Phys. 2009, 81, 739-805.

(61) Bonn, D.; Ross, D. Wetting Transitions. Rep. Prog. Phys. 2001, 64, 1085.

(62) Bergeron, V.; Bonn, D.; Martin, J.; Vovelle, L. Controlling Droplet Deposition with Polymer Additives. Nature 2000, 405, 772775.

(63) Melo-Espinosa, E. A.; Sánchez-Borroto, Y.; Errasti, M.; PilotoRodríguez, R.; Sierens, R.; Roger-Riba, J.; Christopher-Hansen, A. Surface Tension Prediction of Vegetable Oils Using Artificial Neural Networks and Multiple Linear Regression. Energy Procedia 2014, 57, $886-895$.

(64) Joanny, J. F.; de Gennes, P. G. A Model for Contact Angle Hysteresis. J. Chem. Phys. 1984, 81, 552-12.

(65) de Gennes, P. G. Wetting: Statics and Dynamics. Rev. Mod. Phys. $1985,57,827-863$.

(66) Israelachvili, J. N.; Gee, M. L. Contact Angles on Chemically Heterogeneous Surfaces. Langmuir 1989, 5, 288-289. 